\title{
Supersymmetric quantum spin chains and classical integrable systems
}

\author{
Zengo Tsuboi, ${ }^{a}$ Anton Zabrodin ${ }^{b, c, d}$ and Andrei Zotov ${ }^{e, b}$ \\ ${ }^{a}$ Department of Mathematics and Statistics, The University of Melbourne, \\ Royal Parade, Parkville, Victoria 3010, Australia \\ ${ }^{b}$ ITEP, \\ 25 B.Cheremushkinskaya, Moscow 117218, Russia \\ ${ }^{c}$ Institute of Biochemical Physics RAS, \\ 4 Kosygina st., Moscow 119334, Russia \\ ${ }^{d}$ Laboratory of Mathematical Physics, National Research University Higher School of Economics, \\ 20 Myasnitskaya Ulitsa, Moscow 101000, Russia \\ e Steklov Mathematical Institute RAS, \\ 8 Gubkina str., Moscow 119991, Russia \\ E-mail: ztsuboi@yahoo.co.jp, zabrodin@itep.ru, zotov@mi.ras.ru
}

ABSTRACT: For integrable inhomogeneous supersymmetric spin chains (generalized graded magnets) constructed employing $Y(g l(N \mid M))$-invariant $R$-matrices in finite-dimensional representations we introduce the master T-operator which is a sort of generating function for the family of commuting quantum transfer matrices. Any eigenvalue of the master T-operator is the tau-function of the classical mKP hierarchy. It is a polynomial in the spectral parameter which is identified with the 0 -th time of the hierarchy. This implies a remarkable relation between the quantum supersymmetric spin chains and classical manybody integrable systems of particles of the Ruijsenaars-Schneider type. As an outcome, we obtain a system of algebraic equations for the spectrum of the spin chain Hamiltonians.

KEYwords: Lattice Integrable Models, Integrable Hierarchies, Integrable Equations in Physics, Bethe Ansatz

ARXIV EPRINT: 1412.2586 


\section{Contents}

1 Introduction $\quad 2$

2 The master T-operator for supersymmetric spin chains $\quad 7$

2.1 Quantum $R$-matrices $\quad 7$

2.2 Inhomogeneous susy-XXX chains $\quad 9$

2.2.1 T-operators, non-local Hamiltonians and integrals of motion 9

$\begin{array}{ll}\text { 2.2.2 Diagonalization of the T-operator via Bethe ansatz } & 11\end{array}$

$\begin{array}{ll}2.3 \text { The higher T-operators } & 12\end{array}$

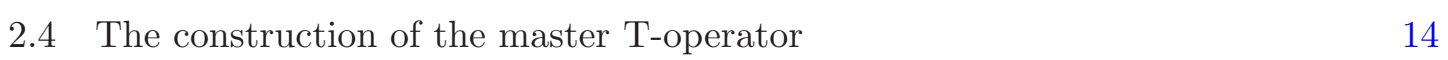

$\begin{array}{ll}2.5 & \text { The master T-operator and the mKP hierarchy } \\ \end{array}$

2.5.1 The bilinear identity for the master T-operator 16

$\begin{array}{ll}\text { 2.5.2 The Baker-Akhiezer functions } & 17\end{array}$

3 From the master T-operator to the classical RS model and back $\quad 19$

3.1 Eigenvalues of the spin chain Hamiltonians as velocities of the RS particles 19

3.2 Lax pair for the RS model from dynamics of poles 20

3.3 The BA function and the master T-operator 22

4 Spectrum of the spin chain Hamiltonians from the classical RS model 23

4.1 Twist parameters as eigenvalues of the Lax matrix 23

4.2 The QC correspondence 24

$\begin{array}{lll}4.3 & \text { Algebraic equations for the spectrum } & 25\end{array}$

5 The QC correspondence via nested Bethe ansatz $\quad 27$

5.1 Bethe ansatz solution for $Y(g l(N \mid M))$ spin chain 27

5.2 The QC correspondence: a direct proof 28

6 Concluding remarks $\quad 30$

$\begin{array}{ll}\text { A The higher T-operators through supercharacters } & 31\end{array}$

B Hamiltonian formulation of the RS model $\quad 32$

C Examples for small values of $L$ and limiting cases 33 


\section{Introduction}

Supersymmetric extensions of quantum integrable spin chains were introduced in $[1,2]$. Such models, called graded magnets in [2], are based on solutions of the graded Yang-Baxter equation (graded analogues of quantum $R$-matrices) in the same way as ordinary integrable spin chains are built from quantum $R$-matrices satisfying the Yang-Baxter $R R R=R R R$ relation. An important class of solutions are $Y(g l(N \mid M))$-invariant $R$-matrices taken in finite-dimensional representations which are simple rational functions of the spectral parameter $x$ :

$$
\mathbf{R}(x)=\mathbf{1} \otimes \mathbf{1}+\frac{\eta}{x} \mathbf{P} .
$$

Here $\mathbf{P} \in \operatorname{End}\left(\mathbb{C}^{N \mid M} \otimes \mathbb{C}^{N \mid M}\right)$ is the graded permutation operator. In this paper, we focus on graded magnets constructed using the $R$-matrices from this class. They are called (generalized) supersymmetric spin chains of the XXX type, or simply susy-XXX spin chains. Their trigonometric analogue is known as the Perk-Schultz model [3].

For our purpose we need inhomogeneous susy-XXX spin chains or the corresponding integrable lattice models of statistical mechanics on inhomogeneous lattices, with quasiperiodic (twisted) boundary conditions. Their quantum monodromy matrices $\mathbf{S}(x)$ are products of the type

$$
\mathbf{S}(x)=\mathbf{R}^{0 L}\left(x-x_{L}\right) \ldots \mathbf{R}^{02}\left(x-x_{2}\right) \mathbf{R}^{01}\left(x-x_{1}\right)\left(\mathbf{g} \otimes \mathbf{1}^{\otimes L}\right)
$$

along the chain, with $x_{i}$ being inhomogeneity parameters assumed to be distinct and $\mathbf{g} \in$ $G L(N \mid M)$ being the twist matrix assumed to be diagonal. (The label 0 corresponds to the auxiliary space, where the product is taken.) The supertrace $\mathbf{T}(x)=\operatorname{str}_{0} \mathbf{S}(x)$ of the quantum monodromy matrix in the auxiliary space is what is called the quantum transfer matrix or the T-operator. The graded Yang-Baxter equation implies that the T-operators commute for all $x$, so $\mathbf{T}(x)$ is a generating function of commuting Hamiltonians $\mathbf{H}_{j}$ :

$$
\mathbf{T}(x)=\operatorname{str} \mathbf{g} \cdot \mathbf{1}^{\otimes L}+\sum_{j=1}^{L} \frac{\eta \mathbf{H}_{j}}{x-x_{j}} .
$$

These Hamiltonians are non-local, i.e., involve interaction between operators on all lattice sites. However, such models still make sense as generalized spin chains with long-range interactions. Alternatively, one may prefer to keep in mind integrable lattice models of statistical mechanics rather than spin chains as such. In either case the final goal of the theory is diagonalization of the T-operators. This is usually achieved by the Bethe ansatz method in one form or another. What we are going to do in this paper is to suggest an alternative approach based on a hidden connection with classical many-body integrable systems explained below.

As an intermediate step, we need to recall that there exists a broader family of commuting T-operators which includes $\mathbf{T}(x)$ as a subset. Using the fusion procedure in the auxiliary space, one can construct an infinite family of commuting T-operators $\mathbf{T}_{\lambda}(x)$ indexed by Young diagrams $\lambda$, with $\mathbf{T}_{\square}(x)=\mathbf{T}(x)$. Following [4], we construct the master T-operator 
as their generating (operator-valued) function of a special form. Let $\mathbf{t}=\left\{t_{1}, t_{2}, t_{3}, \ldots\right\}$ be an infinite set of auxiliary "time variables" and $s_{\lambda}(\mathbf{t})$ be the Schur polynomials. The master T-operator, $\mathbf{T}(x, \mathbf{t})$, for graded magnets ${ }^{1}$ is introduced in the same way as in [4] and subsequent works $[6,7]-[10]$ :

$$
\frac{\mathrm{T}(x, \mathbf{t})}{\mathbf{T}(x, \mathbf{0})}=\sum_{\lambda} \mathbf{T}_{\lambda}(x) s_{\lambda}(\mathbf{t}) .
$$

By construction, this family of operators is commutative for all $x, \mathbf{t}$ and can be simultaneously diagonalized: $\mathrm{T}(x, \mathbf{t})|\Psi\rangle=T(x, \mathbf{t})|\Psi\rangle$. The main fact about the master T-operator, which makes the whole construction interesting, is that the so defined $\mathbf{T}(x, \mathbf{t})$ satisfies the bilinear identity for the classical modified Kadomtsev-Petviashvili (mKP) hierarchy, with $x$ being identified with the "0th time" $t_{0}$ :

$$
\oint_{\mathcal{C}} z^{\left(x-x^{\prime}\right) / \eta} e^{\sum_{k \geq 1}\left(t_{k}-t_{k}^{\prime}\right) z^{k}} \mathbf{T}\left(x, \mathbf{t}-\left[z^{-1}\right]\right) \mathrm{T}\left(x^{\prime}, \mathbf{t}^{\prime}+\left[z^{-1}\right]\right) d z=0
$$

for all $\mathbf{t}, \mathbf{t}^{\prime}, x, x^{\prime}$ and for a properly chosen integration contour. Here $\mathbf{t} \pm\left[z^{-1}\right]:=$ $\left\{t_{k} \pm \frac{1}{k} z^{-k}\right\}$. This means that any eigenvalue $T(x, \mathbf{t})$ of $\mathbf{T}(x, \mathbf{t})$ is a tau-function of the mKP hierarchy. In this way, the commutative algebras of susy-XXX spin chain Hamiltonians, for all possible gradings, appear to be embedded into the infinite integrable hierarchy of non-linear differential-difference equations, the mKP hierarchy [11-13]. This is a further development of the earlier studies [14-17] clarifying the role of classical integrable hierarchies in quantum integrable models.

The next step depends on analytical properties of the eigenvalues $T(x, \mathbf{t})$ as functions of the variable $x$. For finite spin chains each eigenvalue is a polynomial in $x$ of degree $L$ for any $\mathbf{t}$ :

$$
T(x, \mathbf{t})=e^{\operatorname{str} \xi(\mathbf{t}, \mathbf{g})} \prod_{j=1}^{L}\left(x-x_{j}(\mathbf{t})\right) .
$$

The roots depend on the times $\mathbf{t}$. At this point, a surprising link to integrable many-body systems of classical mechanics comes into play. Namely, from the fact that $T(x, \mathbf{t})$ is a tau-function of the mKP hierarchy it follows $[18,19]$ that the roots $x_{i}$ move in the times $t_{k}$ as particles of the Ruijsenaars-Schneider (RS) L-body system [20] subject to the equations of motion corresponding to the $k$ th Hamiltonian $\mathcal{H}_{k}$ of the RS model. For example, the equations of motion for the $t=t_{1}$ flow are

$$
\ddot{x}_{i}=-\sum_{k \neq i} \frac{2 \eta^{2} \dot{x}_{i} \dot{x}_{k}}{\left(x_{i}-x_{k}\right)\left[\left(x_{i}-x_{k}\right)^{2}-\eta^{2}\right]}, \quad i=1, \ldots L .
$$

This link prompts to reformulate the spectral problem for the susy-XXX spin chain Hamiltonians $\mathbf{H}_{j}$ in terms of the integrable model of classical mechanics. It is important to stress that this quantum-classical correspondence (the QC-correspondence) does not depend on

\footnotetext{
${ }^{1} \mathrm{~A}$ preliminary form of the master T-operator for these models appeared in the earlier work [5].
} 
the choice of the grading: all graded magnets are linked to the same RS system of particles. The role of the QC-correspondence in supersymmetric gauge theories and branes was discussed in [21-23].

The RS system is often referred to as an integrable relativistic deformation of the famous Calogero-Moser system. Similarly to the latter, it admits the Lax representation, i.e. the dynamics can be translated into isospectral deformations of a matrix $\mathbf{Z}\left(\left\{x_{i}(t)\right\},\left\{\dot{x}_{i}(t)\right\}\right)$ which is called the Lax matrix. The essence of the QC-correspondence of integrable systems lies in the fact that the spectra of the quantum Hamiltonians $\mathbf{H}_{j}$ are encoded in the Lax matrix $\mathbf{Z}\left(\left\{x_{i}(0)\right\},\left\{\dot{x}_{i}(0)\right\}\right) \equiv \mathrm{Z}_{0}$ for the RS system at $t=0$ after the identifications $x_{i}(0):=x_{i}$ (the inhomogeneity parameters of the spin chain) and $-\eta \dot{x}_{i}(0):=H_{i}$ (the eigenvalues of the quantum Hamiltonians):

$$
\left(\mathrm{Z}_{0}\right)_{i j}=\frac{\eta H_{i}}{x_{j}-x_{i}+\eta} .
$$

Given the $x_{i}$ 's, possible values of the $H_{i}$ 's are determined from the condition that the matrix $Z_{0}$ has a prescribed set of eigenvalues which is a subset of $\left\{g_{1}, g_{2}, \ldots, g_{N+M}\right\}$, where $g_{i}$ are elements of the (diagonal) twist matrix $\mathbf{g}$, taken with certain multiplicities. In this way the spectral problem for the quantum Hamiltonians is reduced to a sort of inverse spectral problem for the Lax matrix.

We give two different proofs of this remarkable correspondence. One (indirect) is through the mKP hierarchy and its polynomial solutions. The other proof (based on the technique developed in [23]) is by a direct computation using the description of the spectrum in terms of the (nested) Bethe ansatz equations. Both proofs are rather technical. It is of value to find a more conceptual proof.

As a corollary, computing the spectral determinant for the RS Lax matrix, we find that the eigenvalues of the quantum Hamiltonians for all XXX spin chains on $L$ sites are encoded in the following system of algebraic equations:

$$
\sum_{1 \leq i_{1}<\ldots<i_{n} \leq L} H_{i_{1}} \ldots H_{i_{n}} \prod_{1 \leq \alpha<\beta \leq n}\left(1-\frac{\eta^{2}}{\left(x_{i_{\alpha}}-x_{i_{\beta}}\right)^{2}}\right)^{-1}=e_{n}\left(g_{1}, \ldots, g_{L}\right), \quad n=1, \ldots, L
$$

Here $e_{n}$ are elementary symmetric polynomials of $L$ parameters $g_{i}: e_{1}=\sum_{i} g_{i}, e_{2}=$ $\sum_{i<j} g_{i} g_{j}$, etc. Identifying them with elements of the twist matrix in a proper way (at $L>N+M$ some $g_{i}$ 's have to be merged), one finds a part of the spectrum $\left(H_{1}, \ldots, H_{L}\right)$ for a particular spin chain among solutions to the system. Other solutions of the same system correspond to some other spin chain. In order to find the full spectrum of a given model, one should solve systems of the form (1.9), where $g_{i}$ 's are taken with different possible multiplicities from a given set. Two points are worth emphasizing:

- These are equations for the spectrum itself, not for any auxiliary parameters like in the Bethe ansatz solution.

- This system does not depend on the grading, so the set of its solutions contain spectra of quantum Hamiltonians for the models with all possible gradings. 
The detailed structure of solutions and their precise correspondence with spectra of particular spin chains is a subject of further study.

From the algebro-geometric point of view, equations (1.9) define a $2 L$-dimensional algebraic variety $\mathbb{S}_{L}$ which can be called the universal spectral variety for spin chains of the XXX type. It contains a comprehensive information about spectra of spin chains on $L$ sites based on the $g l(N \mid M)$ algebras with all possible gradings. The variety $\mathbb{S}_{L}$ given by equations (1.9) is not compact. These equations only define its affine part embedded into the $3 L$-dimensional space with coordinates $\left(H_{1}, \ldots, H_{L} ; x_{1}, \ldots, x_{L} ; g_{1}, \ldots, g_{L}\right)$. Presumably, a proper compactification of the universal spectral variety encodes information about the spectra of homogeneous spin chain Hamiltonians (when $x_{i} \rightarrow 0$ ).

Organization of the paper. In section 2 we recall the construction of the integrable susy-XXX spin chains starting from the quantum $R$-matrices. For our purpose we need a fully inhomogeneous model with twisted boundary conditions. We introduce the nonlocal commuting Hamiltonians, which are the main observables in the system, and such attendant objects like the higher T-operators (transfer matrices). The master T-operator is introduced in section 2.4 as their generating function. In section 2.5 we present the bilinear identity satisfied by the master T-operator which makes it possible to embed the quantum stuff into the context of classical integrable hierarchies of non-linear PDE's. In particular, we define the classical Baker-Akhiezer function in terms of the quantum T-operators.

In section 3 we establish and exploit the link to the RS $L$-body system. The main point here is the reformulation of the eigenvalue problem for the spin chain Hamiltonians in terms of coordinates and velocities of the RS particles. The Lax pair for the RS system is derived from the poles dynamics of the Baker-Akhiezer function in section 3.2.

Section 4 contains some details of the QC correspondence which is based on the identification of the twist parameters with eigenvalues of the Lax matrix for the RS model (section 4.1). In section 4.3 the algebraic equations for eigenvalues of the spin chain Hamiltonians are obtained and the notion of the universal spectral variety is introduced. In section 5 we give a direct proof of the $\mathrm{QC}$ correspondence, using the nested Bethe ansatz solution. In section 6 some unsolved problems are listed.

There are also three appendices. In appendix A we give some technical details needed for deriving the higher T-operators in a more or less explicit form as derivatives of supercharacters. Appendix B is a reference source for the Hamiltonian approach to the RS system. Explicit examples of spin chain spectra for small number of sites $(L=1,2,3)$ and their comparison with solutions to the algebraic equations from section 4.3 are given in appendix C.

Acknowledgments. We thank A. Alexandrov, A. Gorsky, A. Liashyk, V. Kazakov, S. Khoroshkin, I. Krichever and S. Leurent for discussions. The work of Z. Tsuboi is supported by the Australian Research Council. The work of A. Zabrodin is supported in part by RFBR grants 14-02-00627, 12-02-91052-CNRS, 14-01-90405-Ukr and by grant Nsh1500.2014.2 for support of scientific schools. The financial support from the Government of the Russian Federation within the framework of the implementation of the 5-100 Programme Roadmap of the National Research University Higher School of Economics is 
acknowledged. The work of A. Zotov is supported in part by RFBR grants 14-01-00860, 15-51-52031-NNS ${ }_{a}$, by the D. Zimin's fund "Dynasty" and by the Program of RAS "Basic Problems of the Nonlinear Dynamics in Mathematical and Physical Sciences" П19.

The notation. Throughout the paper, we use the following notation.

$\mathfrak{B}$ : any one of the subsets of $\{1,2, \ldots, N+M\}$ with $\operatorname{Card}(\mathfrak{B})=N$.

$\mathfrak{F}$ : the complement set $\mathfrak{F}=\{1,2, \ldots, N+M\} \backslash \mathfrak{B}$

$\mathrm{p}$ : the $\mathbb{Z}_{2}$-grading parameter, $\mathrm{p}(a)=0$ for $a \in \mathfrak{B}$ and $\mathrm{p}(a)=1$ for $a \in \mathfrak{F}$

$\mathbf{e}_{a b}$ : generators of $g l(N \mid M)$ identified with (super)matrix units, $\left(\mathbf{e}_{a b}\right)_{a^{\prime} b^{\prime}}=\delta_{a a^{\prime}} \delta_{b b^{\prime}}$

$\mathbf{v}_{a}$ : orthonormal basis vectors in $\mathbb{C}^{N \mid M}$ such that $\mathbf{e}_{a b} \mathbf{v}_{c}=\delta_{b c} \mathbf{v}_{a}, \mathrm{p}\left(\mathbf{v}_{a}\right)=\mathrm{p}(a)$

g: a diagonal group element of $G L(N \mid M), \mathbf{g}=\operatorname{diag}\left(g_{1}, \ldots, g_{N+M}\right)$

str: the supertrace $\operatorname{str} \mathcal{M}=\sum_{a}(-1)^{\mathrm{p}(a)} \mathcal{M}_{a a}$,

sdet: the superdeterminant $\operatorname{sdet} \mathcal{M}=\exp (\operatorname{str} \log \mathcal{M})$,

$\lambda$ : a Young diagram with rows $\lambda_{1} \geq \lambda_{2} \geq \ldots \geq \lambda_{\ell}>0$

$\lambda^{\prime}$ : the transposed Young diagram ( $\lambda$ reflected in the main diagonal)

1: the identity element in $\operatorname{End}\left(\mathbb{C}^{N \mid M}\right)$ or $\operatorname{End}\left(\mathbb{C}^{L}\right)$

I: the identity operator in the tensor product spaces like $\left(\mathbb{C}^{N \mid M}\right)^{\otimes L}$, etc

O: an operator in $\left(\mathbb{C}^{N \mid M}\right)^{\otimes L}$ or $\mathbb{C}^{L} \otimes\left(\mathbb{C}^{N \mid M}\right)^{\otimes L}$

$\mathbf{O}(x)$ : an operator-valued rational function of $x$

$\mathrm{O}(x)$ : an operator-valued polynomial function of $x$

The $\mathbb{Z}_{2}$-grading of the $g l(N \mid M)$-generators $\mathbf{e}_{a b}$ (identified with the matrix units) is defined as $\mathrm{p}\left(\mathbf{e}_{a b}\right)=\mathrm{p}(a)+\mathrm{p}(b) \bmod 2$. The commutation relations obeyed by the generators are $\mathbf{e}_{a b} \mathbf{e}_{c d}-(-1)^{\mathbf{p}\left(\mathbf{e}_{a b}\right) \mathfrak{p}\left(\mathbf{e}_{c d}\right)} \mathbf{e}_{c d} \mathbf{e}_{a b}=\delta_{b c} \mathbf{e}_{a d}-(-1)^{\mathfrak{p}\left(\mathbf{e}_{a b}\right) \mathfrak{p}\left(\mathbf{e}_{c d}\right)} \delta_{a d} \mathbf{e}_{c b}$.

Any tensor product in this paper is the $\mathbb{Z}_{2}$-graded one. Namely, for any homogeneous operators $\left\{\mathbf{A}_{i}\right\}_{i=1}^{4}$ the tensor product satisfies the relation $\left(\mathbf{A}_{1} \otimes \mathbf{A}_{2}\right)\left(\mathbf{A}_{3} \otimes \mathbf{A}_{4}\right)=$ $(-1)^{\mathbf{p}\left(\mathbf{A}_{2}\right) \mathbf{p}\left(\mathbf{A}_{3}\right)}\left(\mathbf{A}_{1} \mathbf{A}_{3} \otimes \mathbf{A}_{2} \mathbf{A}_{4}\right)$.

We use the notation $\prod_{j=1}^{\stackrel{L}{L}} \mathbf{O}_{j}=\mathbf{O}_{1} \mathbf{O}_{2} \ldots \mathbf{O}_{L}$ and $\prod_{j=1}^{\overleftarrow{L}} \mathbf{O}_{j}=\mathbf{O}_{L} \ldots \mathbf{O}_{2} \mathbf{O}_{1}$ for the ordered product of the operators $\left\{\mathbf{O}_{j}\right\}_{j=1}^{L}$. 


\section{The master T-operator for supersymmetric spin chains}

\subsection{Quantum $R$-matrices}

The simplest $Y(g l(N \mid M))$-invariant $R$-matrix has the form

$$
\mathbf{R}(x)=\mathbf{1} \otimes \mathbf{1}+\frac{\eta}{x} \sum_{a, b=1}^{K}(-1)^{\mathbf{p}(b)} \mathbf{e}_{a b} \otimes \mathbf{e}_{b a} .
$$

Hereafter, we set $K \equiv N+M$. The variable $x$ is the spectral parameter. The extra parameter $\eta$ is not actually essential because it can be eliminated by a rescaling of $x$ (unless one tends $\eta$ to 0 as in the limit to the Gaudin model). The $R$-matrix (2.1) is an operator in the space $\mathbb{C}^{N \mid M} \otimes \mathbb{C}^{N \mid M}$. It can be represented as $\mathbf{R}(x)=\mathbf{1} \otimes \mathbf{1}+\frac{\eta}{x} \mathbf{P}$, where $\mathbf{P}$ is the graded permutation operator given by $\mathbf{P}=\sum_{a, b=1}^{K}(-1)^{\mathbf{p}(b)} \mathbf{e}_{a b} \otimes \mathbf{e}_{b a}$. It acts on homogeneous vectors as follows: $\mathbf{P} \mathbf{x} \otimes \mathbf{y}=(-1)^{\mathrm{p}(\mathbf{x}) \mathrm{p}(\mathbf{y})} \mathbf{y} \otimes \mathbf{x}$.

Having in mind the construction of the spin chain on $L$ sites, one can realize the $R$-matrix as an operator in the space $\mathbb{C}^{N \mid M} \otimes\left(\mathbb{C}^{N \mid M}\right)^{\otimes L}$ :

$$
\mathbf{R}^{0 j}(x)=\mathbf{1} \otimes \mathbf{1}^{\otimes L}+\frac{\eta}{x} \sum_{a, b=1}^{K}(-1)^{\mathrm{p}(b)} \mathbf{e}_{a b} \otimes \mathbf{e}_{b a}^{(j)},
$$

where $\mathbf{e}_{b a}^{(j)}:=\mathbf{1}^{\otimes(j-1)} \otimes \mathbf{e}_{b a} \otimes \mathbf{1}^{\otimes(L-j)}$ for $j \in\{1,2, \ldots, L\} .^{2}$ The first space $\mathbb{C}^{N \mid M}$ labelled by the index 0 is called the auxiliary space while the space $\mathcal{V}=\left(\mathbb{C}^{N \mid M}\right)^{\otimes L}$ is the quantum space of the model. The matrix elements $\left(\mathbf{R}^{0 j}(x)\right)_{a b}$ of the operator $\mathbf{R}^{0 j}(x)$ with respect to the auxiliary space are operators in the quantum space. They are defined by

$$
\mathbf{R}^{0 j}(x)\left(\mathbf{v}_{a} \otimes \mathbf{1}^{\otimes L}\right)=\sum_{b=1}^{K}\left(\mathbf{v}_{b} \otimes \mathbf{1}^{\otimes L}\right)\left(1 \otimes\left(\mathbf{R}^{0 j}(x)\right)_{b a}\right)=\sum_{b=1}^{K} \mathbf{v}_{b} \otimes\left(\mathbf{R}^{0 j}(x)\right)_{b a},
$$

where $\mathbf{v}_{a}$ are orthonormal basis vectors in $\mathbb{C}^{N \mid M}$. From (2.2) we obtain:

$$
\left(\mathbf{R}^{0 j}(x)\right)_{a b}=\delta_{a b} \mathbf{1}^{\otimes L}+(-1)^{\mathbf{p}(a) \mathbf{p}(b)} \frac{\eta}{x} \mathbf{e}_{b a}^{(j)},
$$

where we have used

$$
\left(\mathbf{e}_{a c} \otimes \mathbf{e}_{c a}^{(j)}\right)\left(\mathbf{v}_{b} \otimes \mathbf{1}^{\otimes L}\right)=(-1)^{\mathrm{p}\left(\mathbf{e}_{c a}^{(j)}\right) \mathrm{p}\left(\mathbf{v}_{b}\right)} \mathbf{e}_{a c} \mathbf{v}_{b} \otimes \mathbf{e}_{c a}^{(j)}=(-1)^{(\mathrm{p}(c)+\mathrm{p}(a)) \mathrm{p}(b)} \delta_{c b} \mathbf{v}_{a} \otimes \mathbf{e}_{c a}^{(j)} .
$$

For example, in the $g l(1 \mid 1)$-case with the grading $(\mathrm{p}(1), \mathrm{p}(2))=(0,1)$ the block matrix representation reads

$$
\mathbf{R}^{0 j}(x)=\left(\begin{array}{cc}
\mathbf{I}+\frac{\eta}{x} \mathbf{e}_{11}^{(j)} & \frac{\eta}{x} \mathbf{e}_{21}^{(j)} \\
\frac{\eta}{x} \mathbf{e}_{12}^{(j)} & \mathbf{I}-\frac{\eta}{x} \mathbf{e}_{22}^{(j)}
\end{array}\right)
$$

\footnotetext{
${ }^{2}$ The definition of the graded tensor product implies that $\mathbf{e}_{a b}^{(i)} \mathbf{e}_{c d}^{(j)}=(-1)^{(\mathrm{p}(a)+\mathrm{p}(b))(\mathrm{p}(c)+\mathrm{p}(d))} \mathbf{e}_{c d}^{(j)} \mathbf{e}_{a b}^{(i)}$ for $i \neq j$.
} 
Here $\mathbf{I} \equiv \mathbf{1}^{\otimes L}$. Below we will keep the notation $\mathbf{1}$ for identity elements of $\operatorname{End}\left(\mathbb{C}^{N \mid M}\right)$ and $\operatorname{End}\left(\mathbb{C}^{L}\right)$ and will often write $\mathbf{I}$ for the identity operator in any other spaces involved.

One may also extend the definition of the graded permutation to any two tensor factors of the space $\mathcal{V}$ :

$$
\mathbf{P}_{i j}=\sum_{a, b=1}^{K}(-1)^{\mathbf{p}(b)} \mathbf{e}_{a b}^{(i)} \mathbf{e}_{b a}^{(j)} .
$$

On tensor products of the basis vectors it acts as follows (here $i<j$ ):

$$
\begin{aligned}
\mathbf{P}_{i j}\left(\mathbf{v}_{a_{1}}\right. & \left.\otimes \cdots \otimes \mathbf{v}_{a_{i}} \otimes \cdots \otimes \mathbf{v}_{a_{j}} \otimes \cdots \otimes \mathbf{v}_{a_{L}}\right) \\
& =(-1)^{\mathrm{p}\left(a_{i}\right)+\left(\mathrm{p}\left(a_{i}\right)+\mathrm{p}\left(a_{j}\right)\right) \sum_{k=i}^{j-1} \mathrm{p}\left(a_{k}\right)}\left(\mathbf{v}_{a_{1}} \otimes \cdots \otimes \mathbf{v}_{a_{j}} \otimes \cdots \otimes \mathbf{v}_{a_{i}} \otimes \cdots \otimes \mathbf{v}_{a_{L}}\right) .
\end{aligned}
$$

The aforesaid is related to the $R$-matrix in the evaluation representation of $Y(g l(N \mid M))$ based on the vector representation of $g l(N \mid M)$. More generally, one can consider other irreducible finite-dimensional representations. In this paper we shall restrict our consideration to the class of covariant tensor representations [26-28]. Any partition $\lambda$ (identified with the Young diagram) labels a covariant representation $\pi_{\lambda}$ of the universal enveloping algebra $U(g l(N \mid M))$ if $\lambda_{N+1} \leq M$. For any such representation one can construct an $R$-matrix acting in the tensor product of two spaces, one of which being the representation space $V_{\lambda}$ where the representation $\pi_{\lambda}$ is realized, while the other one is still $\mathbb{C}^{N \mid M}$. We distinguish two $R$-matrices of this type depending on the order of the spaces. One is the $R$-matrix with the auxiliary space $V_{\lambda}$. It has the form

$$
\mathbf{R}_{\lambda}(x)=\mathbf{1} \otimes \mathbf{1}+\frac{\eta}{x} \sum_{a, b=1}^{K}(-1)^{\mathrm{p}(b)} \pi_{\lambda}\left(\mathbf{e}_{a b}\right) \otimes \mathbf{e}_{b a} .
$$

The auxiliary space of the other one is $\mathbb{C}^{N \mid M}$ :

$$
\mathbf{R}^{\lambda}(x)=\mathbf{1} \otimes \mathbf{1}+\frac{\eta}{x} \sum_{a, b=1}^{K}(-1)^{\mathrm{p}(b)} \mathbf{e}_{a b} \otimes \pi_{\lambda}\left(\mathbf{e}_{b a}\right) .
$$

Clearly, $\mathbf{R}_{\square}(x)=\mathbf{R}^{\square}(x)=\mathbf{R}(x)$.

It is convenient to denote

$$
\mathbf{P}_{\lambda}^{0 j}=\sum_{a, b=1}^{K}(-1)^{\mathbf{p}(b)} \pi_{\lambda}\left(\mathbf{e}_{a b}\right) \otimes \mathbf{e}_{b a}^{(j)}, \quad \mathbf{P}_{0 j}^{\lambda}=\sum_{a, b=1}^{K}(-1)^{\mathbf{p}(b)} \mathbf{e}_{a b} \otimes \pi_{\lambda}\left(\mathbf{e}_{b a}^{(j)}\right),
$$

then the $R$-matrix acting non-trivially in the tensor product of the auxiliary space $V_{\lambda}$ and the $j$ th space $\mathbb{C}^{N \mid M}$ is $\mathbf{R}_{\lambda}^{0 j}(x)=\mathbf{I}+\frac{\eta}{x} \mathbf{P}_{\lambda}^{0 j}$ while the $R$-matrix acting nontrivially in the tensor product of the auxiliary space $\mathbb{C}^{N \mid M}$ and the $j$ th space $V_{\Lambda^{(j)}}$ is $\mathbf{R}_{0 j}^{\Lambda^{(j)}}(x)=\mathbf{I}+\frac{\eta}{x} \mathbf{P}_{0 j}^{\Lambda^{(j)}}$. The $R$-matrix $\mathbf{R}^{\lambda}(x)$ obeys the graded Yang-Baxter equation

$$
\mathbf{R}_{12}^{\square}\left(x_{1}-x_{2}\right) \mathbf{R}_{13}^{\lambda}\left(x_{1}-x_{3}\right) \mathbf{R}_{23}^{\lambda}\left(x_{2}-x_{3}\right)=\mathbf{R}_{23}^{\lambda}\left(x_{2}-x_{3}\right) \mathbf{R}_{13}^{\lambda}\left(x_{1}-x_{3}\right) \mathbf{R}_{12}^{\square}\left(x_{1}-x_{2}\right)
$$


and possesses the invariance property

$$
\pi_{\square}(\mathrm{g}) \otimes \pi_{\lambda}(\mathrm{g}) \mathbf{R}^{\lambda}(x)=\mathbf{R}^{\lambda}(x) \pi_{\square}(\mathbf{g}) \otimes \pi_{\lambda}(\mathbf{g})
$$

valid for any $\mathbf{g}$. The graded Yang-Baxter equation for the $R$-matrix $\mathbf{R}_{\lambda}(x)$ reads

$$
\mathbf{R}_{\lambda, \mu}^{12}\left(x_{1}-x_{2}\right) \mathbf{R}_{\lambda}^{13}\left(x_{1}-x_{3}\right) \mathbf{R}_{\mu}^{23}\left(x_{2}-x_{3}\right)=\mathbf{R}_{\mu}^{23}\left(x_{2}-x_{3}\right) \mathbf{R}_{\lambda}^{13}\left(x_{1}-x_{3}\right) \mathbf{R}_{\lambda, \mu}^{12}\left(x_{1}-x_{2}\right),
$$

where $\mathbf{R}_{\lambda, \mu}^{12}(x) \in \operatorname{End}\left(V_{\lambda} \otimes V_{\mu}\right)$ is a yet more general $R$-matrix. Its explicit form is complicated. The invariance property for the $\mathbf{R}_{\lambda}(x)$ is similar to (2.11) with the opposite order of the tensor factors.

\subsection{Inhomogeneous susy-XXX chains}

Here we construct, using the $R$-matrices from the previous subsection, the inhomogeneous integrable susy-XXX spin chains with twisted boundary conditions.

\subsubsection{T-operators, non-local Hamiltonians and integrals of motion}

Let $\mathbf{g} \in G L(N \mid M)$ be a group element represented by a diagonal (super)matrix $\mathbf{g}=$ $\operatorname{diag}\left(g_{1}, g_{2}, \ldots, g_{K}\right)=\sum_{a=1}^{K} g_{a} \mathbf{e}_{a a}$. We call it the twist matrix with the twist parameters $g_{i}$. It is used for the construction of an integrable spin chain with twisted boundary conditions. The T-operator (the transfer matrix) of the inhomogeneous spin chain with twisted boundary conditions is defined by

$$
\mathbf{T}(x)=\operatorname{str}_{0}\left(\mathbf{R}^{0 L}\left(x-x_{L}\right) \ldots \mathbf{R}^{02}\left(x-x_{2}\right) \mathbf{R}^{01}\left(x-x_{1}\right)(\mathbf{g} \otimes \mathbf{I})\right),
$$

where $x_{1}, x_{2}, \ldots, x_{L}$ are inhomogeneity parameters. We assume that they are in general position meaning that $x_{i} \neq x_{j}$ and $x_{i} \neq x_{j} \pm \eta$ for all $i \neq j$. As is known, the Yang-Baxter equation implies that the T-operators with fixed inhomogeneous and twist parameters commute: $\left[\mathbf{T}(x), \mathbf{T}\left(x^{\prime}\right)\right]=0$ for any $x, x^{\prime}$.

The dynamical variables of the model (which we call "spins" in analogy with the rank 1 case) are vectors in the vector representation of $g l(N \mid M)$ realized in the spaces $\mathbb{C}^{N \mid M}$ attached to each site. One can define a set of non-local commuting Hamiltonians $\mathbf{H}_{j}$ as residues of $\mathbf{T}(x)$ at $x=x_{j}$ :

$$
\mathbf{T}(x)=\mathbf{I} \operatorname{str} \mathbf{g}+\sum_{j=1}^{L} \frac{\eta \mathbf{H}_{j}}{x-x_{j}} .
$$

In general, the Hamiltonians $\mathbf{H}_{j}$ imply a long-range interaction involving all spins in the chain (cf. [29]). Their explicit form is

$$
\begin{aligned}
\mathbf{H}_{j} & =\prod_{k=1}^{\overleftarrow{j-1}}\left(\mathbf{I}+\frac{\eta \mathbf{P}_{k j}}{x_{j}-x_{k}}\right) \mathbf{g}^{(j)} \prod_{k=j+1}^{\overleftarrow{L}}\left(\mathbf{I}+\frac{\eta \mathbf{P}_{j k}}{x_{j}-x_{k}}\right) \\
& =\sum_{I \subseteq\{1,2, \ldots, L\} \backslash\{j\}} \eta^{|I|}\left(\prod_{k \in I} \frac{1}{x_{j}-x_{k}}\right)\left(\overleftarrow{\prod_{k \in I, k<j}} \mathbf{P}_{k j}\right) \mathbf{g}^{(j)}\left(\overleftarrow{\prod_{k \in I, k>j}} \mathbf{P}_{j k}\right)
\end{aligned}
$$


where $\mathbf{g}^{(j)}:=\mathbf{1}^{\otimes(j-1)} \otimes \mathbf{g} \otimes \mathbf{1}^{\otimes(L-j)}$ and $\mathbf{P}_{i j}$ is the graded permutation operator (2.5). In the second line, the sum is taken over all subsets $I$ of the set $\{1,2, \ldots, L\} \backslash\{j\}$ including the empty one; $|I| \equiv$ Card $I$.

In addition to the Hamiltonians $\mathbf{H}_{j}$, there are other integrals of motion. It is easy to see that the operators

$$
\mathbf{M}_{a}=\sum_{j=1}^{L} \mathbf{e}_{a a}^{(j)}
$$

referred to as weight operators, commute with the $\mathbf{H}_{i}{ }^{\prime}$ s: $\left[\mathbf{H}_{j}, \mathbf{M}_{a}\right]=0$. Therefore, the eigenstates of the Hamiltonians can be classified according to the eigenvalues $\left(M_{1}, \ldots, M_{K}\right)$ of the weight operators referred to as weights. For example, in the $g l(2)$-case, $M_{1}$ and $M_{2}$ are the numbers of spins with positive and negative $z$-projections respectively.

Let $\mathcal{V}=\left(\mathbb{C}^{N \mid M}\right)^{\otimes L}=\bigoplus_{M_{1}, \ldots, M_{K}} \mathcal{V}\left(\left\{M_{a}\right\}\right)$ be the "weight decomposition" of the quantum space into the direct sum of weight spaces which are eigenspaces of the weight operators with the eigenvalues $M_{a} \in \mathbb{Z}_{\geq 0}, a=1, \ldots, K$. Then any eigenstate of the $\mathbf{H}_{j}$ 's belongs to some weight space $\mathcal{V}\left(\left\{M_{a}\right\}\right)$. The dimension of the weight space $\mathcal{V}\left(\left\{M_{a}\right\}\right)$ is given by

$$
\operatorname{dim} \mathcal{V}\left(\left\{M_{a}\right\}\right)=\frac{L !}{M_{1} ! \ldots M_{K} !} .
$$

In particular, let $1 \leq a_{0} \leq K$ be some fixed index, then the space with $M_{a}=L \delta_{a a_{0}}$ is one-dimensional. It is spanned by the vector $\mathbf{v}_{a_{0}} \otimes \ldots \otimes \mathbf{v}_{a_{0}}$ which is an eigenvector of the Hamiltonians (2.15). Indeed, using (2.6) in the particular case $a_{1}=\ldots=a_{L}=a_{0}$, one can see that

$$
\mathbf{H}_{j}\left(\mathbf{v}_{a_{0}} \otimes \cdots \otimes \mathbf{v}_{a_{0}}\right)=g_{a_{0}} \prod_{k=1, \neq j}^{L}\left(1+\frac{(-1)^{\mathrm{p}\left(a_{0}\right)} \eta}{x_{j}-x_{k}}\right)\left(\mathbf{v}_{a_{0}} \otimes \ldots \otimes \mathbf{v}_{a_{0}}\right) .
$$

The weight operators are not all independent. Since $\sum_{a} \mathbf{e}_{a a}=\mathbf{1}$, we have $\sum_{a} \mathbf{M}_{a}=L \mathbf{I}$ and hence $\sum_{a} M_{a}=L$. Note also that

$$
\sum_{j=1}^{L} \mathbf{H}_{j}=\sum_{j=1}^{L} \mathbf{g}^{(j)}=\sum_{a=1}^{K} g_{a} \mathbf{M}_{a}
$$

so the model has $L+N+M-1$ independent commuting integrals of motion.

For completeness, we give here the definition of the T-operator for a more general inhomogeneous spin chain model with the quantum space $\bigotimes_{j=1}^{L} V_{\Lambda^{(j)}}$ and the auxiliary space $\mathbb{C}^{N \mid M}$. The spin chain is defined by the following data:

- The number of sites, $L$, and the inhomogeneity parameters $x_{i}$ at each site;

- Covariant tensor representations of $g l(N \mid M)$ indexed by the Young diagrams

$$
\Lambda^{(j)}=\left(\Lambda_{1}^{(j)}, \ldots, \Lambda_{K}^{(j)}\right) \in\left(\mathbb{Z}_{\geq 0}\right)^{K}, \quad \Lambda_{1}^{(j)} \geq \Lambda_{2}^{(j)} \geq \ldots \geq \Lambda_{K}^{(j)} \geq 0
$$

assigned to each site $j=1, \ldots, L$; 
- Elements of the diagonal twist matrix $\mathbf{g}=\operatorname{diag}\left(g_{1}, \ldots, g_{K}\right)$ (the twist parameters).

The T-operator

$$
\mathbf{T}^{\Lambda}(x)=\operatorname{str}_{0}\left(\mathbf{R}_{0 L}^{\Lambda^{(L)}}\left(x-x_{L}\right) \ldots \mathbf{R}_{02}^{\Lambda^{(2)}}\left(x-x_{2}\right) \mathbf{R}_{01}^{\Lambda^{(1)}}\left(x-x_{1}\right)(\mathbf{g} \otimes \mathbf{I})\right) .
$$

acts in the space $\bigotimes_{j=1}^{L} V_{\Lambda^{(j)}}$. One may also introduce a set of Hamiltonians $\mathbf{H}_{j}^{\Lambda}$ in the way similar to $(2.14)$ :

$$
\mathbf{T}^{\Lambda}(x)=\mathbf{I} \operatorname{str} \mathbf{g}+\sum_{j=1}^{L} \frac{\eta \mathbf{H}_{j}^{\Lambda}}{x-x_{j}} .
$$

Our main objects of interest will be the T-operator $\mathbf{T}(x)$ and the Hamiltonians $\mathbf{H}_{j}$ of the model with vector representations at the sites corresponding to the choice $\Lambda^{(j)}=$ $(1,0, \ldots, 0)$ for all $j=1, \ldots, L$.

\subsubsection{Diagonalization of the T-operator via Bethe ansatz}

The algebraic form of the nested Bethe ansatz technique for the twisted $Y(g l(n))$ inhomogeneous spin chain [30] can be naturally extended to the $Y(g l(N \mid M))$-case $[2,16,17,31-33]$. The T-operators and the Hamiltonians $\mathbf{H}_{j}^{\Lambda}$ can be diagonalized by this method. Although in what follows we need only the result for the choice $\Lambda^{(j)}=(1,0, \ldots, 0),{ }^{3}$ we give here the general result for future references.

Eigenvalues of the T-operator $\mathbf{T}^{\Lambda}(x)$ are given by

$$
\begin{aligned}
\mathrm{T}^{\Lambda}(x)= & \sum_{b=1}^{K}(-1)^{\mathrm{p}(b)} g_{b} \\
& \times \prod_{k=1}^{L} \frac{x-x_{k}+(-1)^{\mathrm{p}(b)} \eta \Lambda_{b}^{(k)}}{x-x_{k}} \prod_{\gamma=1}^{L_{b-1}} \frac{x-\mu_{\gamma}^{b-1}+(-1)^{\mathrm{p}(b)} \eta}{x-\mu_{\gamma}^{b-1}} \prod_{\gamma=1}^{L_{b}} \frac{x-\mu_{\gamma}^{b}-(-1)^{\mathrm{p}(b)} \eta}{x-\mu_{\gamma}^{b}},
\end{aligned}
$$

The corresponding eigenvalues of the Hamiltonians (2.14) are as follows:

$$
\begin{gathered}
H_{\Lambda, i}=\eta^{-1} \operatorname{Res}_{x=x_{i}} \mathrm{~T}^{\Lambda}(z)=\sum_{b=1}^{K} \Lambda_{b}^{(k)} g_{b} \\
\times \prod_{k \neq i}^{L} \frac{x_{i}-x_{k}+(-1)^{\mathrm{p}(b)} \Lambda_{b}^{(k)} \eta}{x_{i}-x_{k}} \prod_{\gamma=1}^{L_{b-1}} \frac{x_{i}-\mu_{\gamma}^{b-1}+(-1)^{\mathrm{p}(b)} \eta}{x_{i}-\mu_{\gamma}^{b-1}} \prod_{\gamma=1}^{L_{b}} \frac{x_{i}-\mu_{\gamma}^{b}-(-1)^{\mathrm{p}(b)} \eta}{x_{i}-\mu_{\gamma}^{b}} .
\end{gathered}
$$

It is convenient to set $L_{0}=L_{K}=0$. The parameters $\mu_{\alpha}^{b}$ with

$$
\alpha=1, \ldots, L_{b}, \quad b=1, \ldots, K-1, \quad L \geq L_{1} \geq L_{2} \geq \ldots \geq L_{K-1} \geq 0
$$

are Bethe roots. They satisfy the system of Bethe equations which are equivalent to the conditions

$$
\underset{x=\mu_{\alpha}^{b}}{\operatorname{Res}} \mathrm{T}^{\Lambda}(x)=0 \quad \text { for all } \alpha=1, \ldots, L_{b}, \quad b=1, \ldots, K-1 .
$$

\footnotetext{
${ }^{3}$ The Bethe ansatz for trigonometric models closely related to this case was discussed in [34].
} 
The Bethe equations have the form:

$$
\begin{aligned}
& g_{b} \prod_{k=1}^{L} \frac{\mu_{\beta}^{b}-x_{k}+(-1)^{\mathfrak{p}(b)} \Lambda_{b}^{(k)} \eta}{\mu_{\beta}^{b}-x_{k}+(-1)^{\mathrm{p}(b+1)} \Lambda_{b+1}^{(k)} \eta} \prod_{\gamma=1}^{L_{b-1}} \frac{\mu_{\beta}^{b}-\mu_{\gamma}^{b-1}+(-1)^{\mathrm{p}(b)} \eta}{\mu_{\beta}^{b}-\mu_{\gamma}^{b-1}} \\
= & g_{b+1} \prod_{\gamma \neq \beta}^{L_{b}} \frac{\mu_{\beta}^{b}-\mu_{\gamma}^{b}+(-1)^{\mathfrak{p}(b+1)} \eta}{\mu_{\beta}^{b}-\mu_{\gamma}^{b}-(-1)^{\mathrm{p}(b)} \eta} \prod_{\gamma=1}^{L_{b+1}} \frac{\mu_{\beta}^{b}-\mu_{\gamma}^{b+1}-(-1)^{\mathfrak{p}(b+1)} \eta}{\mu_{\beta}^{b}-\mu_{\gamma}^{b+1}} .
\end{aligned}
$$

Later we will specify these general formulae for the highest weights

$$
\Lambda^{(j)}=(1,0, \ldots, 0) \quad \text { for all } j=1, \ldots, L, \text { i.e., } \Lambda_{b}^{(j)}=\delta_{b 1} .
$$

With this choice the first product in the l.h.s. of (2.27) disappears for $b \geq 2$.

\subsection{The higher T-operators}

The $R$-matrix (2.7) allows one to construct a family of T-operators with the more general auxiliary space:

$$
\mathbf{T}_{\lambda}(x)=\operatorname{str}_{V_{\lambda}}\left(\mathbf{R}_{\lambda}^{0 L}\left(x-x_{L}\right) \ldots \mathbf{R}_{\lambda}^{02}\left(x-x_{2}\right) \mathbf{R}_{\lambda}^{01}\left(x-x_{1}\right)\left(\pi_{\lambda}(\mathbf{g}) \otimes \mathbf{I}\right)\right) .
$$

Obviously, $\mathbf{T}_{\square}(x)$ coincides with the T-operator $\mathbf{T}(x)$ introduced previously. The $\mathrm{T}$ operators with fixed inhomogeneous and twist parameters commute with $\mathbf{T}(x)$ and among themselves:

$$
\left[\mathbf{T}_{\lambda}(x), \mathbf{T}_{\mu}\left(x^{\prime}\right)\right]=0
$$

for any $x, x^{\prime}, \lambda, \mu$. At $\lambda=\emptyset$ we put $\mathbf{T}_{\emptyset}(x)$ equal to the identity operator: $\mathbf{T}_{\emptyset}(x)=\mathbf{I}$.

It is clear from (2.7) and (2.29) that at $L=0$ (the empty quantum space) as well as in the limit $x \rightarrow \infty$ for any $L$ the T-operators become equal to the supercharacters $\chi_{\lambda}(\mathbf{g})=\operatorname{str}_{V_{\lambda}}$ g. In what follows we also need the next-to-leading term of the expansion of $\mathbf{T}_{\lambda}(x)$ as $x \rightarrow \infty$. From the definition (2.29) one obtains the expansion

$$
\mathbf{T}_{\lambda}(x)=\chi_{\lambda}(\mathbf{g}) \mathbf{I}+\left.\frac{\eta}{x} \sum_{j=1}^{L} \sum_{a, b}(-1)^{\mathbf{p}(b)} \frac{\partial \chi_{\lambda}\left(e^{\mathbf{e}_{a b}} \mathbf{g}\right)}{\partial \varepsilon}\right|_{\varepsilon=0} \mathbf{e}_{b a}^{(j)}+O\left(1 / x^{2}\right)
$$

Indeed, we have $\mathbf{T}_{\lambda}(x)=\chi_{\lambda}(\mathbf{g}) \mathbf{I}+\frac{\eta}{x} \sum_{j=1}^{L} \operatorname{str}_{V_{\lambda}}\left(\mathbf{P}_{\lambda}^{0 j} \pi_{\lambda}(\mathbf{g})\right)+O\left(1 / x^{2}\right)$ which is converted to the form (2.31) by the following chain of equalities:

$\operatorname{str}_{V_{\lambda}}\left(\mathbf{P}_{\lambda}^{0 j} \pi_{\lambda}(\mathbf{g})\right)=\sum_{a, b}(-1)^{\mathbf{p}(b)} \operatorname{str}_{V_{\lambda}} \pi_{\lambda}\left(\mathbf{e}_{a b} \mathbf{g}\right) \mathbf{e}_{b a}^{(j)}=\left.\sum_{a, b}(-1)^{\mathbf{p}(b)} \frac{\partial}{\partial \varepsilon}\left[\operatorname{str}_{V_{\lambda}} \pi_{\lambda}\left(e^{\varepsilon \mathbf{e}_{a b}} \mathbf{g}\right)\right]\right|_{\varepsilon=0} \mathbf{e}_{b a}^{(j)}$ 
In fact the following explicit expression for the T-operator in terms of the supercharacters is available:

$$
\begin{aligned}
& \mathbf{T}_{\lambda}(x)=\sum_{l=0}^{L} \eta^{l} \sum_{\substack{i_{1}<\ldots<i_{l} a_{1}, \ldots, a_{l} \\
b_{1}, \ldots, b_{l}}}^{L} \prod_{\alpha=1}^{l}\left(\frac{(-1)^{\mathbf{p}\left(b_{\alpha}\right)} \mathbf{e}_{b_{\alpha} a_{\alpha}}^{\left(i_{\alpha}\right)}}{x-x_{i_{\alpha}}} \frac{\partial}{\partial \varepsilon_{\alpha}}\right) \chi_{\lambda}\left(\left.e^{\left.\varepsilon_{l} \mathbf{e}_{a_{l} b_{l}} \ldots e^{\varepsilon_{1} \mathbf{e}_{a_{1} b_{1}}} \mathbf{g}\right)}\right|_{\varepsilon_{\alpha}=0}\right. \\
& =\prod_{l=1}^{\vec{L}}\left(\mathbf{I}+\eta \sum_{a_{l}, b_{l}} \frac{(-1)^{\mathbf{p}\left(b_{l}\right)} \mathbf{e}_{b_{l} a_{l}}^{(l)}}{x-x_{l}} \frac{\partial}{\partial \varepsilon_{l}}\right) \chi_{\lambda}\left(\left.e^{\left.\varepsilon_{l} \mathbf{e}_{a_{l} b_{l}} \ldots e^{\varepsilon_{1} \mathbf{e}_{a_{1} b_{1}}} \mathbf{g}\right)}\right|_{\varepsilon_{l}=0} .\right.
\end{aligned}
$$

The summation over each $a_{\alpha}$ and $b_{\alpha}$ runs from 1 to $K=N+M$. The derivation of (2.32) is sketched in appendix A. ${ }^{4}$

As is known [26], supercharacters of the covariant tensor representations are expressed by the same formulas as for ordinary groups except for traces replaced by supertraces. In particular, the supercharacters are known to satisfy the Jacobi-Trudi identities [36] which have superficially the same form as the ones for the usual characters:

$$
\chi_{\lambda}(\mathbf{g})=\operatorname{det}_{1 \leq i, j \leq \lambda_{1}^{\prime}} \chi_{\lambda_{i}-i+j}(\mathbf{g})=\operatorname{det}_{1 \leq i, j \leq \lambda_{1}} \chi^{\lambda_{i}^{\prime}-i+j}(\mathbf{g}) .
$$

Here $\chi_{k}:=\chi_{(k)}$ (respectively, $\left.\chi^{k}:=\chi_{\left(1^{k}\right)}\right)$ is the character corresponding to the one-row (respectively, one-column) diagram of length $k$.

There exist analogues of these identities for the T-operators depending on the spectral parameter. These are the Cherednik-Bazhanov-Reshetikhin (CBR) determinant formulas sometimes called the quantum Jacobi-Trudi identities:

$$
\mathbf{T}_{\lambda}(x)=\operatorname{det}_{1 \leq i, j \leq \lambda_{1}^{\prime}} \mathbf{T}_{\lambda_{i}-i+j}(x-(j-1) \eta)=\operatorname{det}_{1 \leq i, j \leq \lambda_{1}} \mathbf{T}^{\lambda_{i}^{\prime}-i+j}(x+(j-1) \eta) .
$$

The determinants are well-defined because all the T-operators commute. Similarly to $(2.33), \mathbf{T}_{k}(x):=\mathbf{T}_{(k)}(x)$ and $\mathbf{T}^{k}(x):=\mathbf{T}_{\left(1^{k}\right)}(x)$ are the T-operators corresponding to the one-row and one-column diagrams respectively. There are the following "boundary conditions" for them: $\mathbf{T}_{k}(x)=\mathbf{T}^{k}(x)=0$ if $k<0$ for $N, M \neq 0 ; \mathbf{T}_{k}(x)=0$ if $k<0$ or $k>M$ at $N=0 ; \mathbf{T}^{k}(x)=0$ if $k<0$, or $k>N$ at $M=0$.

For models based on $Y(g l(N))$-invariant $R$-matrices, the quantum Jacobi-Trudi identities follow from resolutions of modules for the Yangian $Y(g l(N))[37,38]$. In the physical literature, they appeared in [39] for $g l(N)$ (see also [40, 41]), in [42, 43] for $g l(N \mid M)$ and in $[44,45]$ for some infinite dimensional representations in the context of AdS/CFT correspondence.

Remarkably, their general form does not depend on the grading, although their matrix elements and eigenvalues of the T-operators do. In fact the assertion that the T-operators for supersymmetric integrable models satisfy the quantum Jacobi-Trudi identities was a

\footnotetext{
${ }^{4}$ In parentheses in the second line one can recognize the (graded) co-derivative operator [35] which is a version of the matrix derivative. It proved to be a valuable technical tool for the proof of the CBR identities and the master T-operator construction. However, in this paper we do not use the co-derivative explicitly.
} 
conjecture in $[42,43]$. A direct proof of this fact was given in [35] for models based on the $Y(g l(N \mid M))$-invariant $R$-matrices, where each "spin" of the chain was assumed to be in the vector representation. ${ }^{5}$ A proof which is independent of the quantum space is only available [46] for models based on the $q$-deformed algebra $U_{q}(s l(2 \mid 1))$ in the case of rectangular Young diagrams.

The eigenvalues of the T-operators (2.29) are rational functions of $x$ with $L$ poles. Another normalization, where they are polynomials in $x$ of degree $L$, is also convenient and even preferable for the link to classical integrable hierarchies. The polynomial form of the T-operators is obtained as follows:

$$
\mathrm{T}_{\lambda}(x)=\prod_{j=1}^{L}\left(x-x_{j}\right) \mathbf{T}_{\lambda}(x) .
$$

In particular for $\lambda=\emptyset$, we have $\mathrm{T}_{\emptyset}(x)=\prod_{j=1}^{L}\left(x-x_{j}\right)$. The CBR-formulas (2.34) in the polynomial normalization acquire the form

$$
\begin{aligned}
& \mathrm{T}_{\lambda}(x)=\left(\prod_{k=1}^{\lambda_{1}^{\prime}-1} \mathrm{~T}_{\emptyset}(x-k \eta)\right)^{-1} \operatorname{det}_{1 \leq i, j \leq \lambda_{1}^{\prime}} \mathrm{T}_{\lambda_{i}-i+j}(x-(j-1) \eta), \\
& \mathrm{T}_{\lambda}(x)=\left(\prod_{k=1}^{\lambda_{1}-1} \mathrm{~T}_{\emptyset}(x-k \eta)\right)^{-1} \operatorname{det}_{1 \leq i, j \leq \lambda_{1}} \mathrm{~T}^{\lambda_{i}^{\prime}-i+j}(x+(j-1) \eta) .
\end{aligned}
$$

\subsection{The construction of the master T-operator}

The master T-operator is a generating function of the T-operators $\mathrm{T}_{\lambda}(x)$ of a special form. It can be introduced in the same way as in [4]. (In an implicit form, the notion of the master T-operator appeared in [5].) For this we should recall the definition of the Schur functions.

Let $\mathbf{t}=\left\{t_{1}, t_{2}, t_{3}, \ldots\right\}$ be an infinite set of complex parameters (we call them times) and $s_{\lambda}(\mathbf{t})$ be the standard Schur functions ( $S$-functions) which can be introduced as

$$
s_{\lambda}(\mathbf{t})=\operatorname{det}_{1 \leq i, j \leq \lambda_{1}^{\prime}} h_{\lambda_{i}-i+j}(\mathbf{t}),
$$

where the polynomials $h_{k}(\mathbf{t})=s_{(k)}(\mathbf{t})$ (the elementary Schur functions) are defined by

$$
e^{\xi(\mathbf{t}, z)}=\sum_{k=0}^{\infty} h_{k}(\mathbf{t}) z^{k}, \quad \xi(\mathbf{t}, z):=\sum_{n=1}^{\infty} t_{k} z^{k}
$$

It is convenient to put $h_{k}(\mathbf{t})=0$ for negative $k$ and $s_{\emptyset}(\mathbf{t})=1$. As is obvious from the definition, the Schur functions are polynomials in the times $t_{i}$. The Schur functions are often regarded as symmetric functions of variables $\xi_{\alpha}$ such that $t_{k}=\frac{1}{k} \sum_{\alpha} \xi_{\alpha}^{k}$.

\footnotetext{
${ }^{5}$ There was a minor gap in the proof given in [35] which was filled in the appendix of [4] for the $g l(N)$ case. The proof for $g l(N \mid M)$ is similar.
} 
The supercharacters can be expressed in terms of the Schur functions ${ }^{6}$ as follows [26]. Set $y_{k}=\frac{1}{k} \operatorname{str} \mathbf{g}^{k}$, where $\operatorname{str} \mathbf{g}^{k}$ is the supertrace of $\mathbf{g}^{k}$ realized in the vector representation as a $K \times K$ diagonal matrix: $\operatorname{str} \mathbf{g}^{k}=\sum_{a=1}^{N+M}(-1)^{\mathbf{p}(a)} g_{a}^{k}$. Then $\chi_{\lambda}(\mathbf{g})=s_{\lambda}(\mathbf{y})$. This is equivalent to the fact that $(\operatorname{sdet}(\mathbf{1}-z \mathbf{g}))^{-1}$ is the generating function for the supercharacters corresponding to one-row diagrams: $(\operatorname{sdet}(\mathbf{1}-z \mathbf{g}))^{-1}=\sum_{k \geq 0} h_{k}(\mathbf{y}) z^{k}$. (For diagonal matrices sdet $\mathbf{g}=\prod_{a} g_{a}^{1-2 \mathrm{p}(a)}$.) For later use we need the following identity for the supercharacters:

$$
\sum_{\lambda} \chi_{\lambda}(\mathbf{g}) s_{\lambda}(\mathbf{t})=\exp \left(\sum_{k \geq 1} t_{k} \operatorname{str} \mathbf{g}^{k}\right)
$$

which is simply the Cauchy-Littlewood identity for the Schur functions [36]. Here and below, the sum $\sum_{\lambda}$ goes over all Young diagrams $\lambda$ including the empty one.

Now we are ready to introduce the master T-operator as an infinite sum over the Young diagrams:

$$
\mathrm{T}(x, \mathbf{t})=\sum_{\lambda} \mathrm{T}_{\lambda}(x) s_{\lambda}(\mathbf{t}) .
$$

It immediately follows from the definition that the T-operators $T_{\lambda}(x)$ can be restored from it by applying the differential operators in the times $t_{i}$ :

$$
\mathrm{T}_{\lambda}(x)=\left.s_{\lambda}(\tilde{\partial}) \mathbf{T}(x, \mathbf{t})\right|_{\mathbf{t}=0},
$$

where $\tilde{\partial}:=\left\{\partial_{t_{1}}, \frac{1}{2} \partial_{t_{2}}, \frac{1}{3} \partial_{t_{3}}, \ldots\right\} . \quad$ In particular, $\mathbf{T}_{\emptyset}(x)=\mathbf{T}(x, \mathbf{0})=\prod_{j=1}^{L}\left(x-x_{j}\right) \mathbf{I}$ and $\mathrm{T}_{\square}(x)=\left.\partial_{t_{1}} \mathbf{T}(x, \mathbf{t})\right|_{\mathbf{t}=0}$, so that the T-operator $\mathbf{T}(x)=\mathbf{T}_{(1)}(x)=\mathbf{T}_{\square}(x)$ (2.13) is expressed as the logarithmic derivative of the master T-operator:

$$
\mathbf{T}(x)=\left.\partial_{t_{1}} \log \mathbf{T}(x, \mathbf{t})\right|_{\mathbf{t}=0} .
$$

Using (2.31) and the Cauchy-Littlewood identity (2.39), one can derive the following expansion of the master T-operator as $x \rightarrow \infty$ :

$$
\frac{\mathrm{T}(x, \mathbf{t})}{\mathrm{T}_{\emptyset}(x)}=e^{\operatorname{str} \xi(\mathbf{t}, \mathbf{g})}\left(\mathbf{I}+\frac{\eta}{x} \sum_{j=1}^{L} \sum_{k \geq 1} k t_{k}\left(\mathbf{g}^{(j)}\right)^{k}+O\left(1 / x^{2}\right)\right) .
$$

Note that the sign factor $(-1)^{\mathrm{p}(b)}$ coming from the definition of the $\mathbf{P}_{\lambda}^{0 j}(2.9)$ cancels against the one coming from the supertrace, so the two leading terms do not actually depend on the grading (except for the supertrace in the common factor). More generally, using (2.32)

\footnotetext{
${ }^{6}$ This expression is equivalent to the one in terms of the so-called supersymmetric Schur functions (SS-functions) [47] which are symmetric functions of two sets of variables, $\left\{g_{a}\right\}_{a \in \mathfrak{B}}$ and $\left\{g_{b}\right\}_{b \in \mathfrak{F}}$.
} 
and the Cauchy-Littlewood identity one arrives, in a similar way, to the following explicit expression for the master T-operator:

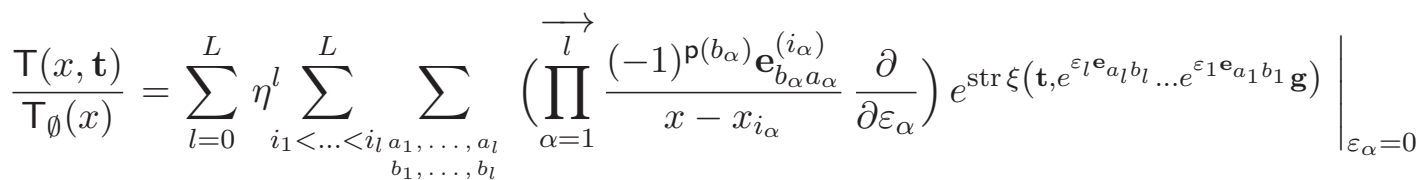

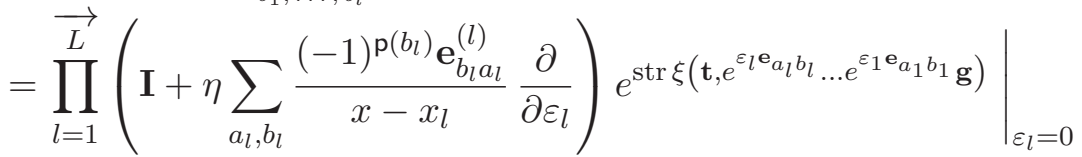

Given $z \in \mathbb{C}$, we will use the standard notation $\mathbf{t} \pm\left[z^{-1}\right]$ for the following special shift of the time variables:

$$
\mathbf{t} \pm\left[z^{-1}\right]:=\left\{t_{1} \pm z^{-1}, t_{2} \pm \frac{1}{2} z^{-2}, t_{3} \pm \frac{1}{3} z^{-3}, \ldots\right\} .
$$

As we shall see below, $\mathbf{T}\left(x, \mathbf{t} \pm\left[z^{-1}\right]\right)$ regarded as functions of $z$ with fixed $x, \mathbf{t}$ play an important role. Here we only note that equation (2.41) implies that $\mathrm{T}\left(x, \mathbf{0} \pm\left[z^{-1}\right]\right)$ are the generating series for the T-operators corresponding to the one-row and one-column diagrams respectively:

$$
\mathrm{T}\left(x,\left[z^{-1}\right]\right)=\sum_{s=0}^{\infty} z^{-s} \mathrm{~T}_{s}(x), \quad \mathrm{T}\left(x,-\left[z^{-1}\right]\right)=\sum_{a=0}^{\infty}(-z)^{-a} \mathbf{T}^{a}(x) .
$$

\subsection{The master T-operator and the mKP hierarchy}

\subsubsection{The bilinear identity for the master T-operator}

The main property of the master T-operator which provides a remarkable link to the theory of classical non-linear integrable equations and their hierarchies is given by the following statement.

Theorem 2.1 The master T-operator (2.40) satisfies the bilinear identity for the mKP hierarchy:

$$
\oint_{\mathcal{C}} z^{\left(x-x^{\prime}\right) / \eta} e^{\xi\left(\mathbf{t}-\mathbf{t}^{\prime}, z\right)} \mathrm{T}\left(x, \mathbf{t}-\left[z^{-1}\right]\right) \mathrm{T}\left(x^{\prime}, \mathbf{t}^{\prime}+\left[z^{-1}\right]\right) d z=0
$$

for all $\mathbf{t}, \mathbf{t}^{\prime}, x$ and $x^{\prime}$. The integration contour $\mathcal{C}$ encircles the cut $[0, \infty]$ between 0 and $\infty$ and does not enclose any singularities coming from the $\mathrm{T}$-factors.

The bilinear identity has superficially the same form as the one for the master T-operator for the ordinary (non-supersymmetric) spin chains associated with $Y(g l(N))$ [4] and can be proved in a similar way. The proof is based on the CBR formulas (2.36) or (2.37). The definition of the master T-operator (2.40) can be interpreted as the expansion of the taufunction in Schur polynomials [11, 48, 49]. The functional relations for quantum transfer matrices [37-43] are then the Plücker-like relations for coefficients of the expansion. ${ }^{7}$

\footnotetext{
${ }^{7}$ It is pertinent to note possible generalizations of this picture. There are functional relations [44, 50] and their Wronskian-like determinant solutions $[51,52]$ related to infinite-dimensional representations of
} 
The bilinear identity (2.46) is a source of various bilinear Hirota equations for the master T-operator. For example, setting $x^{\prime}=x-\eta, \mathbf{t}^{\prime}=\mathbf{t}-\left[z_{1}^{-1}\right]-\left[z_{2}^{-1}\right]$, we obtain the 3 -term difference Hirota equation

$$
\begin{gathered}
z_{2} \boldsymbol{\top}\left(x+\eta, \mathbf{t}-\left[z_{2}^{-1}\right]\right) \mathbf{\top}\left(x, \mathbf{t}-\left[z_{1}^{-1}\right]\right)-z_{1} \mathbf{\top}\left(x+\eta, \mathbf{t}-\left[z_{1}^{-1}\right]\right) \mathbf{\top}\left(x, \mathbf{t}-\left[z_{2}^{-1}\right]\right) \\
+\left(z_{1}-z_{2}\right) \mathbf{\top}(x+\eta, \mathbf{t}) \mathbf{\top}\left(x, \mathbf{t}-\left[z_{1}^{-1}\right]-\left[z_{2}^{-1}\right]\right)=0
\end{gathered}
$$

which is in fact equivalent to the bilinear identity (see [53]).

\subsubsection{The Baker-Akhiezer functions}

Let $T(x, \mathbf{t})$ be any eigenvalue of the master T-operator. As it follows from $(2.46)$, it is a tau-function of the mKP hierarchy. It is then natural to incorporate other key ingredients of the soliton theory. The most important for us are the Baker-Akhiezer (BA) function and its adjoint. In what follows we refer to both as the BA functions. They are defined as

$$
\begin{gathered}
\psi(x, \mathbf{t} ; z)=z^{x / \eta} e^{\xi(\mathbf{t}, z)} \frac{T\left(x, \mathbf{t}-\left[z^{-1}\right]\right)}{T(x, \mathbf{t})}, \\
\psi^{*}(x, \mathbf{t} ; z)=z^{-x / \eta} e^{-\xi(\mathbf{t}, z)} \frac{T\left(x, \mathbf{t}+\left[z^{-1}\right]\right)}{T(x, \mathbf{t})} .
\end{gathered}
$$

We are going to also consider the operator-valued BA functions $\hat{\psi}(x, \mathbf{t} ; z)$ defined by the same formulas with $T(x, \mathbf{t})$ substituted by $\mathrm{T}(x, \mathbf{t})$. Since these operators commute for all $x, \mathbf{t}$, the operator $\hat{\psi}(x, \mathbf{t} ; z)$ is well-defined.

According to the definition of the master T-operator, $\mathrm{T}\left(x, \mathbf{t} \mp\left[z^{-1}\right]\right)$ is an infinite series in $z^{-1}$. From (2.44) one can see that this series converges to a rational function of $z$ for any $x, \mathbf{t}$ if $|z|>\max \left\{\left|g_{1}\right|,\left|g_{2}\right|, \ldots,\left|g_{K}\right|\right\}$. Explicitly, we obtain:

$$
\begin{aligned}
\mathrm{T}\left(x, \mathbf{t} \mp\left[z^{-1}\right]\right)= & \prod_{l=1}^{\vec{L}}\left(\left(x-x_{l}\right) \mathbf{I}+\eta \sum_{a_{l}, b_{l}}(-1)^{\mathbf{p}\left(b_{l}\right)} \mathbf{e}_{b_{l} a_{l}}^{(l)} \frac{\partial}{\partial \varepsilon_{l}}\right) \\
& \times\left.\left\{\left[\operatorname{sdet}\left(\mathbf{1}-z^{-1} \mathbf{g}_{\varepsilon_{L}, \ldots, \varepsilon_{1}}^{a_{L} b_{L}, \ldots, a_{1} b_{1}}\right)\right]^{ \pm 1} e^{\operatorname{str} \xi\left(\mathbf{t}, \mathbf{g}_{\varepsilon_{L}, \ldots, \varepsilon_{1}}^{a_{L} b_{L}, \ldots, a_{1} b_{1}}\right)}\right\}\right|_{\varepsilon_{l}=0},
\end{aligned}
$$

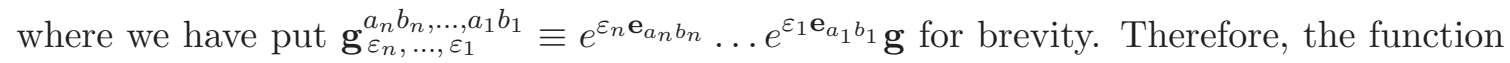
$z^{-x / \eta} e^{-\xi(\mathbf{t}, z)} \psi(x, \mathbf{t} ; z)\left(\right.$ resp. $\left.z^{x / \eta} e^{\xi(\mathbf{t}, z)} \psi^{*}(x, \mathbf{t} ; z)\right)$ is a rational function of $z$ with poles at the points $z=g_{a}$ (the eigenvalues of the matrix $\mathbf{g}$ ) for $a \in \mathfrak{F}$ (resp., $a \in \mathfrak{B}$ ) of at least

$\overline{g l}(N \mid M)$. The solutions are given by changing the expansion point of the generating function for the Toperators. Therefore, there is a possibility that the master T-operator (2.40) is still relevant for such systems after a sort of analytic continuation. In this paper, we consider only covariant tensor representations of $g l(N \mid M)$. There are also contravariant and mixed representations whose characters are labelled by a pair of Young diagrams. It is an interesting open problem whether the corresponding master T-operator is a tau-function of any hierarchy of soliton equations (like the 2D Toda lattice). 
first order. It can be also derived ${ }^{8}$ from (2.50) that

$$
\lim _{z \rightarrow 0} z^{ \pm(N-M)} \mathbf{T}\left(x, \mathbf{t} \mp\left[z^{-1}\right]\right)=(-1)^{N-M}(\operatorname{sdet} \mathbf{g})^{ \pm 1} \mathbf{T}(x \pm \eta, \mathbf{t}) .
$$

The left hand side is to be understood as the analytic continuation to the point $z=0$ of the analytic function (rational in our case) defined by the series which converges in a neighbourhood of infinity.

Since any eigenvalue $T(x, \mathbf{t})$ is a polynomial in $x$, the functions $z^{-x / \eta} \psi$ and $z^{x / \eta} \psi^{*}$, regarded as functions of $x$, are rational functions with $L$ zeros and $L$ poles which are simple in general position. From (2.40), (2.35) and (2.29), using the Cauchy-Littlewood identity, or directly from (2.50), we conclude that

$$
\lim _{x \rightarrow \infty} \frac{T\left(x, \mathbf{t} \mp\left[z^{-1}\right]\right)}{T(x, \mathbf{t})}=\frac{e^{\operatorname{str} \xi\left(\mathbf{t} \mp\left[z^{-1}\right], \mathbf{g}\right)}}{e^{\operatorname{str} \xi(\mathbf{t}, \mathbf{g})}}=e^{\operatorname{str} \xi\left(\mp\left[z^{-1}\right], \mathbf{g}\right)}=\left[\operatorname{sdet}\left(\mathbf{1}-z^{-1} \mathbf{g}\right)\right]^{ \pm 1}
$$

hence

$$
\begin{aligned}
& \lim _{x \rightarrow \infty} z^{-x / \eta} e^{-\xi(\mathbf{t}, z)} \psi(x, \mathbf{t} ;, z)=z^{-N+M} \operatorname{sdet}(z \mathbf{1}-\mathbf{g}), \\
& \lim _{x \rightarrow \infty} z^{x / \eta} e^{\xi(\mathbf{t}, z)} \psi^{*}(x, \mathbf{t} ; z)=z^{N-M}(\operatorname{sdet}(z \mathbf{1}-\mathbf{g}))^{-1} .
\end{aligned}
$$

The (operator-valued) functions $\hat{\psi}(x, z):=\hat{\psi}(x, \mathbf{0} ; z)$ and $\hat{\psi}^{*}(x, z):=\hat{\psi}^{*}(x, \mathbf{0} ; z)$, as well as the corresponding eigenvalues, are called stationary BA functions. Their explicit form directly follows from (2.50):

$$
\begin{gathered}
z^{-x / \eta} \hat{\psi}(x, z)=\left.\sum_{l=0}^{L} \eta^{l} \sum_{\substack{i_{1}<\ldots<i_{l} a_{1}, \ldots, a_{l} \\
b_{1}, \ldots, b l}}^{L}\left(\prod_{\alpha=1}^{l} \frac{(-1)^{\mathrm{p}\left(b_{\alpha}\right)} \mathbf{e}_{b_{\alpha} a_{\alpha}}^{\left(i_{\alpha}\right)}}{x-x_{i_{\alpha}}} \frac{\partial}{\partial \varepsilon_{\alpha}}\right) \operatorname{sdet}\left(\mathbf{1}-z^{-1} \mathbf{g}_{\varepsilon_{l}, \ldots, \varepsilon_{1}}^{a_{l} b_{l}, \ldots, a_{1} b_{1}}\right)\right|_{\varepsilon_{\alpha}=0}, \\
z^{x / \eta} \hat{\psi}^{*}(x, z)=\left.\sum_{l=0}^{L} \eta^{l} \sum_{\substack{i_{1}<\ldots<i_{l a_{1}, \ldots, a_{l}} b_{1}, \ldots, b_{l} \\
b_{1}}}\left(\prod_{\alpha=1}^{l} \frac{(-1)^{\mathrm{p}\left(b_{\alpha}\right)} \mathbf{e}_{b_{\alpha} a_{\alpha}}^{\left(i_{\alpha}\right)}}{x-x_{i_{\alpha}}} \frac{\partial}{\partial \varepsilon_{\alpha}}\right)\left[\operatorname{sdet}\left(\mathbf{1}-z^{-1} \mathbf{g}_{\varepsilon_{l}, \ldots, \varepsilon_{1}}^{a_{l} b_{l}, \ldots, a_{1} b_{1}}\right)\right]^{-1}\right|_{\varepsilon_{\alpha}=0} .
\end{gathered}
$$

8 The main underlying statement is that

$$
\begin{aligned}
& \left.\left(x \mathbf{I}+\eta \sum_{a, b}(-1)^{\mathrm{p}(b)} \mathbf{e}_{b a}^{(l)} \frac{\partial}{\partial \varepsilon}\right)\left[\operatorname{sdet}\left(e^{\varepsilon \mathbf{e}_{a b}} \mathbf{g}\right)\right]^{ \pm 1} \Phi(\varepsilon)\right|_{\varepsilon=0} \\
= & {\left.[\operatorname{sdetg}]^{ \pm 1}\left((x \pm \eta) \mathbf{I}+\eta \sum_{a, b}(-1)^{\mathfrak{p}(b)} \mathbf{e}_{b a}^{(l)} \frac{\partial}{\partial \varepsilon}\right) \Phi(\varepsilon)\right|_{\varepsilon=0} }
\end{aligned}
$$

for any $\mathbf{g} \in G L(M \mid N)$ and any $\Phi \in \operatorname{End}\left(V_{l+1} \otimes V_{l+2} \otimes \ldots \otimes V_{L}\right)$ (here $\left.V_{i} \cong \mathbb{C}^{N \mid M}\right)$. It immediately follows from the Leibniz rule and the identity

$$
\left.\frac{\partial}{\partial \varepsilon}\left[\operatorname{sdet}\left(e^{\varepsilon \mathbf{e}_{a b}} \mathbf{g}\right)\right]^{ \pm 1}\right|_{\varepsilon=0}=\operatorname{str}\left(\mathbf{e}_{a b}\right)[\operatorname{sdet} \mathbf{g}]^{ \pm 1}=(-1)^{\mathbf{p}(b)} \delta_{a b}[\operatorname{sdet} \mathbf{g}]^{ \pm 1}
$$

which is easy to check. 
In particular, we have the expansion of $\hat{\psi}(x, z)$ as $|x| \rightarrow \infty$ :

$$
z^{-x / \eta} \hat{\psi}(x, z)=\operatorname{sdet}\left(\mathbf{1}-z^{-1} \mathbf{g}\right)\left(\mathbf{I}-\frac{\eta}{x} \sum_{j=1}^{L} \sum_{a=1}^{K} \frac{g_{a} \mathbf{e}_{a a}^{(j)}}{z-g_{a}}+O\left(1 / x^{2}\right)\right)
$$

(we need it in the next section).

For calculations in the next section we also need the following general properties of the BA functions of the mKP hiertarchy.

a) They obey the differential-difference equations of the form

$$
\begin{aligned}
\partial_{t_{1}} \psi(x, \mathbf{t} ; z) & =\psi(x+\eta, \mathbf{t} ; z)+V(x, \mathbf{t}) \psi(x, \mathbf{t} ; z), \\
-\partial_{t_{1}} \psi^{*}(x, \mathbf{t} ; z) & =\psi^{*}(x-\eta, \mathbf{t} ; z)+V(x-\eta, \mathbf{t}) \psi^{*}(x, \mathbf{t} ; z)
\end{aligned}
$$

(the linear problems), where $V(x, \mathbf{t})=\partial_{t_{1}} \log \frac{T(x+\eta, \mathbf{t})}{T(x, \mathbf{t})}$ (see, e.g., $\left.[12,13]\right)$;

b) They obey the relation

$$
\partial_{t_{m}} \log \frac{T(x+\eta, \mathbf{t})}{T(x, \mathbf{t})}=\operatorname{res}_{\infty}\left(\psi(x, \mathbf{t} ; z) \psi^{*}(x+\eta, \mathbf{t} ; z) z^{m} \mathrm{~d} z\right)
$$

where the residue is normalized as $\operatorname{res}_{\infty} z^{-1} \mathrm{~d} z=1$. It can be derived from $(2.46)$ and (2.51) in the same way as in [9].

\section{From the master T-operator to the classical RS model and back}

\subsection{Eigenvalues of the spin chain Hamiltonians as velocities of the RS particles}

As we already mentioned in the previous section, the eigenvalues of the master T-operator are polynomials in the spectral parameter $x$ of degree $L$ :

$$
T(x, \mathbf{t})=e^{\operatorname{str} \xi(\mathbf{t}, \mathbf{g})} \prod_{k=1}^{L}\left(x-x_{k}(\mathbf{t})\right) .
$$

The roots have their own dynamics in the times. The very fact that $T(x, \mathbf{t})$ is a tau-function of the mKP hierarchy implies $[18,19]$ that the roots $x_{i}$ move in the time $t_{1}$ as particles of the RS $L$-body system [20]. Moreover, their motion in the higher times $t_{k}$ is the same as motion of the RS particles caused by the higher Hamiltonian flows of the RS system (see $[9,19]$ which extend the methods developed by Krichever [54, 55] and Shiota [56]). We have $T(x, \mathbf{0})=T_{\emptyset}(x)=\prod_{k=1}^{L}\left(x-x_{k}\right)$, where $x_{k}=x_{k}(\mathbf{0})$. This means that

(i) The inhomogeneity parameters $x_{k}$ of the spin chain should be identified with initial positions $x_{k}(\mathbf{0})$ of the RS particles. 
With the help of (2.42) we can write:

$$
\frac{T_{\square}(x)}{T_{\emptyset}(x)}=\left.\partial_{t_{1}} \log T(x, \mathbf{t})\right|_{\mathbf{t}=0}=\operatorname{str} \mathbf{g}-\sum_{k=1}^{L} \frac{\dot{x}_{k}(\mathbf{0})}{x-x_{k}},
$$

where $\dot{x}_{k}(\mathbf{0}):=\left.\partial_{t_{1}} x_{k}(\mathbf{t})\right|_{\mathbf{t}=0}$. Comparing this with (2.14), we find:

$$
\dot{x}_{k}(\mathbf{0})=-\eta H_{k}
$$

where $H_{k}$ is an eigenvalue of $\mathbf{H}_{k}$. Therefore, in addition to (i) we conclude that

(ii) The eigenvalues $H_{k}$ of the susy-XXX spin chain Hamiltonians are expressed through the initial velocities of the RS particles as $H_{i}=-\dot{x}_{i}(\mathbf{0}) / \eta$.

In other words, any point in the phase space of the $L$-body RS system with coordinates $\left\{x_{i}, \dot{x}_{i}\right\}$ corresponds to an eigenstate, with the eigenvalues $H_{i}=-\dot{x}_{i} / \eta$, of the Hamiltonians of the susy-XXX spin chain on $L$ sites with the inhomogeneity parameters $x_{i}$.

This unexpected connection between quantum spin chains and the classical RS model was pointed out in [4] as a corollary of the Hirota bilinear equations for the master Toperator. A similar relation between quantum Hamiltonians in the Gaudin model and velocities of particles in the classical Calogero-Moser model was found in [57, 58] using different methods (see also $[59,60]$ for further developments). ${ }^{9}$ The message of the present paper is that the identifications (i) and (ii) are in fact independent of the grading: their form is the same for all spin chains of the XXX type associated with any (super)algebra $g l(N \mid M)$ including the ordinary algebras $g l(N \mid 0)=g l(N)$.

\subsection{Lax pair for the RS model from dynamics of poles}

To make the correspondence "quantum spin chains $\leftrightarrow$ classical RS systems" (the QC correspondence) complete, we need the Lax matrix for the RS model. At this stage the set-up is exactly the same as in [9]. Here we repeat the main formulas with some comments skipping the details.

Below we will derive equations of motion for the $t_{1}$-dynamics of the $x_{i}$ 's using Krichever's method $[54,55]$, the starting point of which is the linear problem $(2.57)$ for the BA function. Essentially, the derivation is not specific to the master $T$-operator case but only depends on the polynomial form of the tau-function.

One can derive equations of motion for the $x_{i}$ 's performing the pole expansion of the linear problem (2.57). It is convenient to denote $t_{1}=t$ and put all higher times equal to 0 because they are irrelevant for this derivation. Below in this section we often write simply $t$ instead of $\mathbf{t}$. According to (2.48), the general form of $\psi$ as a function of $x$ is

$$
\psi(x, t ; z)=z^{x / \eta} e^{t z}\left(c_{0}(z)+\sum_{j=1}^{L} \frac{c_{j}(z, t)}{x-x_{j}(t)}\right),
$$

\footnotetext{
${ }^{9}$ In $[6,7]$ it was obtained from the master T-operator construction for the Gaudin model.
} 
where $c_{0}(z)=\operatorname{sdet}\left(\mathbf{1}-z^{-1} \mathbf{g}\right)$ (see (2.52)). One should substitute it into the linear equation (2.57) with

$$
V(x, t)=\partial_{t} \log \frac{T(x+\eta, t)}{T(x, t)}=\sum_{k=1}^{L}\left(\frac{\dot{x}_{k}}{x-x_{k}}-\frac{\dot{x}_{k}}{x-x_{k}+\eta}\right), \quad x_{k}=x_{k}(t)
$$

and cancel all the poles at $x=x_{i}$ and $x=x_{i}-\eta$ (possible poles of the second order cancel automatically). This yields an overdetermined system of linear equations for the coefficients $c_{i}$ :

$$
\left\{\begin{array}{l}
(z \mathbf{1}-\mathrm{Z}) \vec{c}=c_{0}(z) \dot{\mathrm{X}} \overrightarrow{1} \\
\dot{\vec{c}}=\mathrm{G} \vec{c}
\end{array}\right.
$$

where $\vec{c}=\left(c_{1}, c_{2}, \ldots, c_{L}\right)^{\mathrm{t}}, \overrightarrow{1}=(1,1, \ldots, 1)^{\mathrm{t}}$ are $L$-component vectors and the $L \times L$ matrices $\mathbf{X}=\mathbf{X}(t), \mathbf{Z}=\mathbf{Z}(t), \mathbf{G}=\mathrm{G}(t)$ are defined by their matrix elements as follows:

$$
\begin{aligned}
& \mathrm{X}_{i j}=x_{i} \delta_{i j}, \quad \mathrm{Z}_{i j}=\frac{\dot{x}_{i}}{x_{i}-x_{j}-\eta} \\
& \mathrm{G}_{i j}=\left(\sum_{k \neq i} \frac{\dot{x}_{k}}{x_{i}-x_{k}}-\sum_{k \neq i} \frac{\dot{x}_{k}}{x_{i}-x_{k}+\eta}\right) \delta_{i j}+\left(\frac{\dot{x}_{i}}{x_{i}-x_{j}}-\frac{\dot{x}_{i}}{x_{i}-x_{j}-\eta}\right)\left(1-\delta_{i j}\right) .
\end{aligned}
$$

The explicit form of the matrix $G$ is not used in what follows. As is easy to check, the matrix $[\mathrm{X}, \mathrm{Z}]-\mathrm{Z}$ has rank 1 . More precisely, these matrices satisfy the commutation relation

$$
[\mathrm{X}, \mathrm{Z}]=\eta \mathrm{Z}+\dot{\mathrm{X}} \mathrm{E}
$$

where $\mathrm{E}=\overrightarrow{1} \otimes \overrightarrow{1}^{\mathrm{t}}$ is the $L \times L$ matrix of rank 1 with all entries equal to 1 . As a consequence of this commutation relation, we mention the identity $\overrightarrow{1}^{t} Z^{k} \dot{X} \overrightarrow{1}=-\eta \operatorname{tr} Z^{k+1}$ which holds for any $k \geq 0$ (see [9]).

The compatibility condition of the problems (3.6) is the Lax equation

$$
\dot{\mathrm{Z}}=[\mathrm{G}, \mathrm{Z}]
$$

which is equivalent to the equations of motion

$$
\ddot{x}_{i}=-\sum_{k \neq i} \frac{2 \eta^{2} \dot{x}_{i} \dot{x}_{k}}{\left(x_{i}-x_{k}\right)\left[\left(x_{i}-x_{k}\right)^{2}-\eta^{2}\right]}, \quad i=1, \ldots, L .
$$

This dynamical system called the RS model is sometimes referred to as the relativistic deformation of the Calogero-Moser model, the parameter $\eta$ being the inverse "velocity of light". The Hamiltonian formulation is given in the appendix. The integrability of the RS model follows from the Lax representation. The matrix $Z$ is the Lax matrix. As it follows from (3.10), the time evolution preserves its spectrum, i.e., the coefficients $\mathcal{J}_{k}$ of the characteristic polynomial

$$
\operatorname{det}(z \mathbf{1}-\mathrm{Z}(t))=\sum_{k=0}^{L} \mathcal{J}_{k} z^{L-k}
$$


are integrals of motion. Equivalently, one can say that eigenvalues of the Lax matrix are integrals of motion.

For completeness, we also present here the linear problem for coefficients of the adjoint BA function

$$
\psi^{*}(x, t ; z)=z^{-x / \eta} e^{-t z}\left(c_{0}^{-1}(z)+\sum_{j=1}^{L} \frac{c_{j}^{*}(z, t)}{x-x_{j}(t)}\right) .
$$

As a counterpart of (3.6), we get, using the equations of motion,

$$
\left\{\begin{array}{l}
\vec{c}^{* t} \dot{\mathbf{X}}^{-1}(z \mathbf{1}-\mathrm{Z})=-c_{0}^{-1}(z) \overrightarrow{1}^{\mathrm{t}} \\
\partial_{t}\left(\vec{c}^{* \mathrm{t}} \dot{\mathbf{X}}^{-1}\right)=-\vec{c}^{* \mathrm{t}} \dot{\mathbf{X}}^{-1} \mathrm{G} .
\end{array}\right.
$$

Here $\vec{c}^{* \mathrm{t}}=\left(c_{1}^{*}, c_{2}^{*}, \ldots, c_{L}^{*}\right)$ and $\overrightarrow{1}^{\mathrm{t}}=(1,1, \ldots, 1)$ (note that $\overrightarrow{1}^{\mathrm{t}} \mathrm{G}=0$ ). Regarding these equations as overdetermined linear problems for the (co)vector $\vec{c}^{* t} \dot{\mathbf{X}}^{-1}$, one comes to the same Lax equation (3.10) as their compatibility condition. The adjoint linear problems (3.14), together with general relation (2.59), are used for the extension of the time dynamics of the $x_{i}$ 's to the whole hierarchy, as it has been done in $[9,19]$, see appendix B.

\subsection{The BA function and the master T-operator}

The solution for the vector $\vec{c}$ reads $\vec{c}(z, t)=c_{0}(z)(z \mathbf{1}-\mathrm{Z}(t))^{-1} \dot{\mathrm{X}} \overrightarrow{1}$. The BA function $\psi$ is then given by the formula

$$
\psi=c_{0}(z) z^{x / \eta} e^{t z}\left(1+\overrightarrow{1}^{\mathrm{t}}(x \mathbf{1}-\mathbf{X})^{-1}(z \mathbf{1}-\mathbf{Z})^{-1} \dot{\mathbf{X}} \overrightarrow{1}\right) .
$$

Similar formulas can be obtained for the adjoint vector $\vec{c}^{* \mathrm{t}}$ and the adjoint BA function:

$$
\begin{aligned}
\vec{c}^{* \mathrm{t}}(z, t) & =-c_{0}^{-1}(z) \overrightarrow{1}^{\mathrm{t}}(z \mathbf{1}-\mathrm{Z}(t))^{-1} \dot{\mathrm{X}}, \\
\psi^{*} & =c_{0}^{-1}(z) z^{-x / \eta} e^{-t z}\left(1-\overrightarrow{1}^{\mathrm{t}}(z \mathbf{1}-\mathrm{Z})^{-1}(x \mathbf{1}-\mathbf{X})^{-1} \dot{\mathrm{X}} \overrightarrow{1}\right) .
\end{aligned}
$$

It is easy to see that for non-zero values of the higher times the BA functions are given by the same formulas with the factor $e^{ \pm t z}$ substituted by $e^{ \pm \xi(\mathbf{t}, z)}$. Writing $\overrightarrow{1}^{\mathrm{t}} \mathrm{A} \overrightarrow{1}=\operatorname{tr}(\mathrm{AE})$ and using the commutation relation (3.9), one can represent these expressions as ratios of determinants: ${ }^{10}$

$$
\begin{aligned}
\psi(x, \mathbf{t} ; z) & =\operatorname{sdet}\left(\mathbf{1}-z^{-1} \mathbf{g}\right) z^{x / \eta} e^{\xi(\mathbf{t}, z)} \frac{\operatorname{det}[(x \mathbf{1}-\mathbf{X})(z \mathbf{1}-\mathbf{Z})-\eta \mathbf{Z}]}{\operatorname{det}(x \mathbf{1}-\mathbf{X}) \operatorname{det}(z \mathbf{1}-\mathbf{Z})}, \\
\psi^{*}(x, \mathbf{t} ; z) & =\left[\operatorname{sdet}\left(\mathbf{1}-z^{-1} \mathbf{g}\right)\right]^{-1} z^{-x / \eta} e^{-\xi(\mathbf{t}, z)} \frac{\operatorname{det}[(z \mathbf{1}-\mathbf{Z})(x \mathbf{1}-\mathbf{X})+\eta \mathbf{Z}]}{\operatorname{det}(x \mathbf{1}-\mathbf{X}) \operatorname{det}(z \mathbf{1}-\mathbf{Z})} .
\end{aligned}
$$

In particular, the stationary BA functions are given by

$$
\begin{aligned}
\psi(x, z) & =\operatorname{sdet}\left(\mathbf{1}-z^{-1} \mathbf{g}\right) z^{x / \eta} \frac{\operatorname{det}\left[\left(x \mathbf{1}-\mathbf{X}_{0}\right)\left(z \mathbf{1}-\mathbf{Z}_{0}\right)-\eta \mathbf{Z}_{0}\right]}{\operatorname{det}\left(x \mathbf{1}-\mathbf{X}_{0}\right) \operatorname{det}\left(z \mathbf{1}-\mathbf{Z}_{0}\right)}, \\
\psi^{*}(x, z) & =\left[\operatorname{sdet}\left(\mathbf{1}-z^{-1} \mathbf{g}\right)\right]^{-1} z^{-x / \eta} \frac{\operatorname{det}\left[\left(z \mathbf{1}-\mathbf{Z}_{0}\right)\left(x \mathbf{1}-\mathbf{X}_{0}\right)+\eta \mathbf{Z}_{0}\right]}{\operatorname{det}\left(x \mathbf{1}-\mathbf{X}_{0}\right) \operatorname{det}\left(z \mathbf{1}-\mathbf{Z}_{0}\right)} .
\end{aligned}
$$

\footnotetext{
${ }^{10}$ The order of the factors $x \mathbf{1}-\mathrm{X}$ and $z \mathbf{1}-\mathrm{Z}$ under the determinant upstairs is actually not important because of the identity $\operatorname{det}\left(A B+A_{1}\right)=\operatorname{det}\left(B A+A_{1}\right)$ valid for any matrices $A, B, A_{1}$ such that $\left[A, A_{1}\right]=0$.
} 
Hereafter, we use the notation $\mathrm{X}_{0}=\mathrm{X}(\mathbf{0}), \mathrm{Z}_{0}=\mathrm{Z}(\mathbf{0})$. Using (2.48), one can obtain from (3.17) an explicit determinant formula for eigenvalues of the master $T$-operator:

$$
T(x, \mathbf{t})=e^{\operatorname{str} \xi(\mathbf{t}, \mathbf{g})} \operatorname{det}\left(x \mathbf{1}-\mathbf{X}_{0}+\sum_{k \geq 1} k t_{k} \mathbf{Z}_{0}^{k}\right) .
$$

Formulas (3.17) and (3.21) are not new in the context of classical integrable hierarchies (see, e.g., [19, 56, 61]). The new observation is the close connection with quantum spin chains. It is important to stress that we can understand (3.19) and (3.21) in the operator sense, i.e. as expressions for the quantum operators $\hat{\psi}(x, z), \mathbf{T}(x, \mathbf{t})$ in terms of the matrices $\left(\mathbf{X}_{0}\right)_{i j}=x_{i} \delta_{i j} \mathbf{I},\left(\mathbf{Z}_{0}\right)_{i j}=\frac{\eta \mathbf{H}_{i}}{x_{j}-x_{i}+\eta}$. The latter is the Lax matrix $\mathbf{Z}_{0}$ with the operatorvalued entries given by equation (3.7) with the substitution (3.3).

To summarize, we have derived equations of motion of the RS model for roots of the master T-operator, together with the Lax representation. Then, embedding the initial quantum problem into the context of the classical RS model, we have obtained explicit operator expressions for the BA function and the master T-operator in terms of the quantum spin chain Hamiltonians. In the next section we show how one can reformulate the spectral problem for the quantum Hamiltonians in terms of an inverse spectral problem for the RS Lax matrix.

\section{Spectrum of the spin chain Hamiltonians from the classical RS model}

\subsection{Twist parameters as eigenvalues of the Lax matrix}

The expansion of the stationary operator-valued BA function $\hat{\psi}$ at large $|x|$ has the same form as in the $g l(N)$ case [9] except for the overall super-determinant. In fact we have two different (but equivalent) expressions for $\hat{\psi}$ : (2.54) and (3.19). Let us compare their large $|x|$ expansions. The large $|x|$ expansion of (2.54) is given by (2.56):

$$
\hat{\psi}(x, z)=c_{0}(z) z^{x / \eta}\left(\mathbf{I}-\frac{\eta}{x} \sum_{j=1}^{L} \sum_{a=1}^{K} \frac{g_{a} \mathbf{e}_{a a}^{(j)}}{z-g_{a}}+O\left(x^{-2}\right)\right),
$$

where $g_{a}$ are the twist parameters. The expansion of (3.19) is

$$
\hat{\psi}(x, z)=c_{0}(z) z^{x / \eta}\left(1-\frac{\eta}{x} \operatorname{tr} \frac{\mathrm{Z}_{0}}{z \mathbf{1}-\mathrm{Z}_{0}}+O\left(x^{-2}\right)\right) .
$$

Equating the $O(1 / x)$ terms of the two expansions leads to the relation

$$
\operatorname{tr} \frac{\mathrm{Z}_{0}}{z \mathbf{1}-\mathrm{Z}_{0}}=\sum_{i} \sum_{a} \frac{\mathbf{e}_{a a}^{(i)} g_{a}}{z-g_{a}}
$$

which has to be valid identically. Let us stress that its left hand side is well-defined because the entries of the matrix $Z_{0}$ are commuting operators. Using the identity $\operatorname{tr}(z \mathbf{1}-\mathrm{A})^{-1}=$ $\partial_{z} \log \operatorname{det}(z \mathbf{1}-\mathrm{A})$ valid for any matrix $\mathrm{A}$, we integrate (4.1) to obtain

$$
\operatorname{det}\left(z \mathbf{1}-\mathbf{Z}_{0}\right)=\prod_{a=1}^{K}\left(z-g_{a}\right)^{\mathbf{M}_{a}}
$$


where $\mathbf{M}_{a}$ are the weight operators (2.17). Since the time evolution is an isospectral deformation, the same is true for the Lax matrix $\mathbf{Z}(\mathbf{t})$ for any values of the times. We see that $\mathbf{M}_{a}$ is the "operator multiplicity" of the eigenvalue $g_{a}$. In the weight space $\mathcal{V}\left(\left\{M_{a}\right\}\right)$ the multiplicities become equal to the $M_{a}$ 's. The conclusion is:

- The Lax matrix Z has eigenvalues $g_{a}$ with multiplicities $M_{a} \geq 0$ such that $M_{1}+\ldots+$ $M_{N}=L$.

Our next goal is to formulate the QC correspondence ${ }^{11}$ between the spin chains and the RS model.

\subsection{The QC correspondence}

Consider the Lax matrix $Z_{0}$ of the $L$-particle RS model, where the inverse "velocity of light", $\eta$, is identified with the parameter $\eta$ introduced in the quantum $R$-matrix (2.2), and the initial coordinates and velocities of the particles are identified, respectively, with the inhomogeneity parameters $x_{i}$ and eigenvalues of the Hamiltonians $H_{i}$ through $\dot{x}_{i}=-\eta H_{i}$ :

$$
\mathrm{Z}_{0}=\left(\begin{array}{ccccc}
H_{1} & \frac{\eta H_{1}}{x_{2}-x_{1}+\eta} & \frac{\eta H_{1}}{x_{3}-x_{1}+\eta} & \cdots & \frac{\eta H_{1}}{x_{L}-x_{1}+\eta} \\
\frac{\eta H_{2}}{x_{1}-x_{2}+\eta} & H_{2} & \frac{\eta H_{2}}{x_{3}-x_{2}+\eta} & \cdots & \frac{\eta H_{2}}{x_{L}-x_{2}+\eta} \\
\vdots & \vdots & \vdots & \ddots & \vdots \\
\frac{\eta H_{L}}{x_{1}-x_{L}+\eta} & \frac{\eta H_{L}}{x_{2}-x_{L}+\eta} & \frac{\eta H_{L}}{x_{3}-x_{L}+\eta} & \cdots & H_{L}
\end{array}\right) .
$$

According to the conclusion of the previous subsection, we claim that if the $H_{i}$ 's are eigenvalues of the Hamiltonians of the spin chain in the weight space $\mathcal{V}\left(\left\{M_{a}\right\}\right) \subset \mathcal{V}$, then

$$
\operatorname{Spec}\left(\mathbf{Z}_{0}\right)=(\underbrace{g_{1}, \ldots, g_{1}}_{M_{1}}, \underbrace{g_{2}, \ldots, g_{2}}_{M_{2}}, \ldots \underbrace{g_{K}, \ldots, g_{K}}_{M_{K}}) .
$$

Equivalently, let $\mathcal{H}_{j}=\operatorname{tr}\left(Z_{0}\right)^{j}$ be the higher integrals of motion of the RS model (see appendix B), then their level set is defined by $\mathcal{H}_{j}=\sum_{a=1}^{K} M_{a} g_{a}^{j}$. In general, the matrix $\mathrm{Z}_{0}$ with multiple eigenvalues is not diagonalizable and contains Jordan cells.

One can also say that the eigenstates of the quantum Hamiltonians correspond to the intersection points of two Lagrangian submanifolds in the phase space of the RS model. One of them is the hyperplane defined by fixing all the coordinates $x_{i}$ while the other one is the Lagrangian submanifold obtained by fixing values of the $L$ independent integrals of motion in involution $\mathcal{H}_{k}, k=1, \ldots, L$. In general, there are many intersection points

\footnotetext{
11 The QC correspondence can be traced back to [62], where joint spectra of some commuting finitedimensional operators were linked to the classical Toda chain.
} 
numbered by a finite set $\mathfrak{I}$, with coordinates, say $\left(x_{1}, \ldots, x_{L}, p_{1}^{(\alpha)}, \ldots, p_{L}^{(\alpha)}\right), \alpha \in \mathfrak{I}$. The values of $p_{j}^{(\alpha)}$ give, through equation (B.2), the spectrum of $\mathbf{H}_{j}$ :

$$
H_{j}^{(\alpha)}=e^{-\eta p_{j}^{(\alpha)}} \prod_{k=1, \neq j} \frac{x_{j}-x_{k}+\eta}{x_{j}-x_{k}} .
$$

However, we can not claim that all the intersection points correspond to the energy levels of the Hamiltonians for a given spin chain. The examples elaborated below in appendix $\mathrm{C}$ suggest that there are intersection points that do not correspond to energy levels of a particular spin chain with a fixed grading. Instead, they correspond to spectra of the Hamiltonians for spin chains with other possible gradings.

Summarizing, we claim that the spectral problem for the non-local inhomogeneous susy-XXX spin chain Hamiltonians $\mathbf{H}_{j}$ in the subspace $\mathcal{V}\left(\left\{M_{a}\right\}\right)$ is closely linked to the following inverse spectral problem for the RS Lax matrix $Z_{0}$ of the form (4.3). Let us fix the spectrum of the matrix $Z_{0}$ to be (4.4), where $g_{1}, \ldots, g_{K}$ are eigenvalues of the (diagonal) twist matrix $\mathbf{g}$. Then we ask what is the set of possible values of the $H_{j}$ 's allowed by these constraints. The eigenvalues $H_{j}$ of the quantum Hamiltonians are contained in this set.

\subsection{Algebraic equations for the spectrum}

The characteristic polynomial of the matrix (4.3) can be found explicitly using the simple fact from the linear algebra that the coefficient in front of $z^{L-k}$ in the polynomial $\operatorname{det}_{L \times L}(z \mathbf{1}+\mathrm{A})$ equals the sum of all diagonal $k \times k$ minors of the matrix A. All such minors can be found using the decomposition $\mathbf{Z}_{0}=-\mathrm{HQ}$, where $\mathbf{H}=\operatorname{diag}\left(H_{1}, H_{2}, \ldots, H_{L}\right)$ and

$$
\mathrm{Q}_{i j}=\frac{\eta}{x_{i}-x_{j}-\eta}
$$

is the Cauchy matrix, and the explicit expression for the determinant of the Cauchy matrix:

$$
\operatorname{det}_{1 \leq i, j \leq n} \frac{\eta}{x_{i}-x_{j}-\eta}=(-1)^{n} \prod_{1 \leq i<j \leq n}\left(1-\frac{\eta^{2}}{\left(x_{i}-x_{j}\right)^{2}}\right)^{-1} .
$$

The result is:

$$
\operatorname{det}_{L \times L}\left(z \mathbf{1}-\mathrm{Z}_{0}\right)=\operatorname{det}_{L \times L}(z \mathbf{1}+\mathrm{HQ})=\sum_{n=0}^{L} \mathcal{J}_{n} z^{L-n}
$$

where

$$
\mathcal{J}_{n}=(-1)^{n} \sum_{1 \leq i_{1}<\ldots<i_{n} \leq L} H_{i_{1}} \ldots H_{i_{n}} \prod_{1 \leq \alpha<\beta \leq n}\left(1-\frac{\eta^{2}}{\left(x_{i_{\alpha}}-x_{i_{\beta}}\right)^{2}}\right)^{-1} .
$$

In particular, the highest coefficient is given by the following simple formula:

$$
\mathcal{J}_{L}=(-1)^{L} H_{1} H_{2} \ldots H_{L} \prod_{1 \leq i<j \leq L}\left(1-\frac{\eta^{2}}{\left(x_{i}-x_{j}\right)^{2}}\right)^{-1} \text {. }
$$

Let us point out that the integrals $\mathcal{H}_{k}$ introduced in the previous section are connected with the integrals $\mathcal{J}_{k}$ by the Newton's formula [36]: $\sum_{k=0}^{L} \mathcal{J}_{L-k} \mathcal{H}_{k}=0$ (we have set $\mathcal{H}_{0}=$ $\left.\operatorname{tr}(\mathrm{Z})^{0}=L\right)$. 
Combining (4.4) and (4.7), we see that the eigenvalues $H_{i}$ of the inhomogeneous susyXXX Hamiltonians can be found from the system of polynomial equations

$$
\sum_{1 \leq i_{1}<\ldots<i_{n} \leq L} H_{i_{1}} \ldots H_{i_{n}} \prod_{1 \leq \alpha<\beta \leq n}\left(1-\frac{\eta^{2}}{\left(x_{i_{\alpha}}-x_{i_{\beta}}\right)^{2}}\right)^{-1}=C_{n}\left(\left\{M_{a}\right\}\right),
$$

where $n=1,2, \ldots, L$ and

$$
\begin{aligned}
C_{n}\left(\left\{M_{a}\right\}\right) & =\frac{1}{2 \pi i} \oint_{|z|=1} \prod_{a=1}^{K}\left(1+z g_{a}\right)^{M_{a}} z^{-n-1} d z \\
& =\sum_{\substack{n_{1}, \ldots, n_{K} \in \mathbb{Z}_{\geq 0} \\
\sum_{j=1}^{K} n_{j}=n}}\left(\begin{array}{c}
M_{1} \\
n_{1}
\end{array}\right) \ldots\left(\begin{array}{c}
M_{K} \\
n_{K}
\end{array}\right) \prod_{a=1}^{K} g_{a}^{n_{a}} .
\end{aligned}
$$

There are $L$ equations for $L$ unknown quantities $H_{1}, \ldots, H_{L}$. Examples for small values of $L$ are given in the appendix.

Here we point out some simple general properties of these equations.

1. This system does not depend on $N \mid M$ and the grading parameters and is also invariant under the following transformations: a) $\eta \rightarrow-\eta$, b) $\left\{x_{i}\right\} \rightarrow\left\{-x_{i}\right\}$, c) $\left\{H_{i}\right\} \rightarrow\left\{-H_{i}\right\}$ simultaneously with $\left\{g_{a}\right\} \rightarrow\left\{-g_{a}\right\}$.

2. For $M_{a}=L \delta_{a a_{1}}$ (in this case $C_{n}=\frac{L !}{n !(L-n) !} g_{a_{1}}^{n}$ ) there are two distinguished solutions

$$
H_{j}=g_{a_{1}} \prod_{k=1, \neq j}^{L}\left(1 \pm \frac{\eta}{x_{j}-x_{k}}\right)
$$

which give the eigenvalues of the Hamiltonians on the vector $\left(\mathbf{v}_{a_{1}}\right)^{\otimes L}$. The two choices of sign correspond to two possible values of the grading parameter $\mathrm{p}\left(a_{1}\right)$.

3. Assume that $L \leq K$ and fix $\left\{a_{1}, \ldots, a_{L}\right\} \subseteq\{1, \ldots, K\}$ such that all the $a_{i}$ 's are distinct. Set $M_{a}=\sum_{i=1}^{L} \delta_{a a_{i}}$, then the right hand sides of equations (4.8) are elementary symmetric polynomials ${ }^{12} e_{k}\left(g_{a_{1}}, \ldots, g_{a_{L}}\right)$ of the twist parameters $g_{a_{1}}, \ldots, g_{a_{L}}$. Then the system of equations (4.8) has $L$ ! solutions (counted with multiplicities). Indeed, at $\eta=0$ the system is just

$$
e_{n}\left(H_{1}, \ldots, H_{L}\right)=e_{n}\left(g_{a_{1}}, \ldots, g_{a_{L}}\right), \quad n=1, \ldots, L
$$

All solutions of this system are given by all possible permutations of the set $\left(g_{a_{1}}, \ldots g_{a_{L}}\right)$ containing $L$ elements.

\footnotetext{
${ }^{12}$ The elementary symmetric polynomials are defined by means of the generating function as follows:

$$
\prod_{i=1}^{\mathcal{N}}\left(1+y_{i} z\right)=\sum_{k=0}^{\mathcal{N}} e_{k}\left(y_{1}, \ldots, y_{\mathcal{N}}\right) z^{k}
$$
}


The detailed structure of solutions to (4.8) and their correspondence with spectra of particular spin chains is a subject of further study. Some examples are discussed in appendix C.

One can consider the system (4.8) with the right hand sides being "in general position" meaning that there are $L$ twist parameters $g_{i}$ which are all distinct. This is the generic situation from which all other possible cases can be obtained by merging some of the twist parameters. The generic system (4.8) contains $L$ equations of the form

$$
\sum_{1 \leq i_{1}<\ldots<i_{n} \leq L} H_{i_{1}} \ldots H_{i_{n}} \prod_{1 \leq \alpha<\beta \leq n}\left(1-\frac{\eta^{2}}{\left(x_{i_{\alpha}}-x_{i_{\beta}}\right)^{2}}\right)^{-1}=e_{n}\left(g_{1}, \ldots, g_{L}\right) .
$$

The complete information about spectra of the Hamiltonians for $L$-site spin chains based on all (super)algebras of the type $g l(N \mid M)$ is contained in the universal spectral variety

$$
\mathbb{S}_{L}=\left\{\left(H_{1}, \ldots H_{L} ; x_{1}, \ldots, x_{L} ; g_{1}, \ldots, g_{L}\right) \mid \text { Equations (4.12) }\right\} .
$$

It is a $2 L$-dimensional affine variety embedded into $\mathbb{C}^{3 L}$. The spectra of the Hamiltonians for particular spin chains are obtained by intersecting with the hyperplanes with fixed values of $x_{i}$ 's and $g_{i}$ 's. The variety $\mathbb{S}_{L}$ is not compact. We anticipate that a proper compactification of the universal spectral variety encodes information about the spectra of Hamiltonians for spin chains when some or all $x_{i}$ 's coalesce.

\section{The QC correspondence via nested Bethe ansatz}

In this section we give a direct proof of the QC correspondence based on the nested Bethe ansatz solution to the susy-XXX spin chains.

\subsection{Bethe ansatz solution for $Y(g l(N \mid M))$ spin chain}

Here we specify the general nested Bethe ansatz results (2.23), (2.27) for the spin chain with vector representations at the sites. The eigenstates of the T-operator $\mathbf{T}(x)$ are obtained from the reference state $\left(\mathbf{v}_{1}\right)^{\otimes L}$ with $M_{a}=L \delta_{a 1}$ by action of creation operators. In the weight space $\mathcal{V}\left(M_{1}, \ldots, M_{K}\right)$ with $M_{1}=L-L_{1}, M_{2}=L_{1}-L_{2}, M_{3}=L_{2}-L_{3}, \ldots$, $M_{K-1}=L_{K-2}-L_{K-1}, M_{K}=L_{K-1}$ such that $L_{1} \geq L_{2} \geq \ldots \geq L_{K}$ the eigenvalues of $\mathbf{T}(x)$ are given by the formula

$$
\begin{aligned}
\mathrm{T}(x)= & (-1)^{\mathrm{p}(1)} g_{1} \prod_{l=1}^{L} \frac{x-x_{l}+(-1)^{\mathrm{p}(1)} \eta}{x-x_{l}} \prod_{\gamma=1}^{L_{1}} \frac{x-\mu_{\gamma}^{1}-(-1)^{\mathrm{p}(1)} \eta}{x-\mu_{\gamma}^{1}} \\
& +\sum_{b=2}^{K}(-1)^{\mathrm{p}(b)} g_{b} \prod_{\alpha=1}^{L_{b-1}} \frac{x-\mu_{\alpha}^{b-1}+(-1)^{\mathrm{p}(b)} \eta}{x-\mu_{\alpha}^{b-1}} \prod_{\gamma=1}^{L_{b}} \frac{x-\mu_{\gamma}^{b}-(-1)^{\mathrm{p}(b)} \eta}{x-\mu_{\gamma}^{b}}
\end{aligned}
$$

where the parameters $\mu_{\gamma}^{b}$ (the Bethe roots) obey the system of Bethe equations

$$
\begin{aligned}
& g_{b} \prod_{k=1}^{L} \frac{\mu_{\beta}^{b}-x_{k}+\delta_{b 1}(-1)^{\mathrm{p}(b)} \eta}{\mu_{\beta}^{b}-x_{k}} \prod_{\gamma=1}^{L_{b-1}} \frac{\mu_{\beta}^{b}-\mu_{\gamma}^{b-1}+(-1)^{\mathrm{p}(b)} \eta}{\mu_{\beta}^{b}-\mu_{\gamma}^{b-1}} \\
& \quad=g_{b+1} \prod_{\gamma \neq \beta}^{L_{b}} \frac{\mu_{\beta}^{b}-\mu_{\gamma}^{b}+(-1)^{\mathrm{p}(b+1)} \eta}{\mu_{\beta}^{b}-\mu_{\gamma}^{b}-(-1)^{\mathrm{p}(b)} \eta} \prod_{\gamma=1}^{L_{b+1}} \frac{\mu_{\beta}^{b}-\mu_{\gamma}^{b+1}-(-1)^{\mathrm{p}(b+1)} \eta}{\mu_{\beta}^{b}-\mu_{\gamma}^{b+1}} .
\end{aligned}
$$


Here $b$ runs from 1 to $K-1=N+M-1$ and the convention $L_{0}=L_{K}=0$ is implied. Note that the first product in the r.h.s. of (5.2) disappears if $(-1)^{\mathrm{p}(b)+\mathrm{p}(b+1)}=-1$. The Bethe equations are equivalent to the conditions that $\mathrm{T}(x)$ given by (5.1) is regular at the points $x=\mu_{\beta}^{b}$ for all $\beta=1, \ldots, L_{b}, \quad b=1, \ldots, K-1$. The corresponding eigenvalues of the Hamiltonians $\mathbf{H}_{i}$ are

$$
H_{i}\left(\left\{x_{i}\right\}_{L},\left\{\mu_{\alpha}^{1}\right\}_{L_{1}}, g_{1}\right)=g_{1} \prod_{k=1}^{L} \frac{x_{i}-x_{k}+(-1)^{\mathrm{p}(1)} \eta}{x_{i}-x_{k}} \prod_{\gamma=1}^{L_{1}} \frac{x_{i}-\mu_{\gamma}^{1}-(-1)^{\mathrm{p}(1)} \eta}{x_{i}-\mu_{\gamma}^{1}}
$$

where $\left\{x_{i}\right\}_{L}$ emphasizes the dependence on $L$ variables $x_{i}\left(\left\{x_{i}\right\}_{L}\right.$ means $\left\{x_{i}\right\}_{i=1}^{L}$, in particular, $\left.\left\{x_{i}\right\}_{0}=\emptyset\right)$ and similarly for $\left\{\mu_{\alpha}^{1}\right\}_{L_{1}}$.

Example: $N+M=2$. In the weight space $\mathcal{V}\left(M_{1}, M_{2}\right)$ with $M_{1} \geq M_{2}$ the Bethe equations are:

$$
\begin{array}{r}
g l(2 \mid 0): \quad g_{1} \prod_{k=1}^{L} \frac{\mu_{\alpha}^{1}-x_{k}+\eta}{\mu_{\alpha}^{1}-x_{k}}=g_{2} \prod_{\gamma \neq \alpha}^{M_{2}} \frac{\mu_{\alpha}^{1}-\mu_{\gamma}^{1}+\eta}{\mu_{\alpha}^{1}-\mu_{\gamma}^{1}-\eta}, \\
g l(1 \mid 1) \text { with } \quad(\mathrm{p}(1), \mathrm{p}(2))=(0,1): \quad g_{1} \prod_{k=1}^{L} \frac{\mu_{\alpha}^{1}-x_{k}+\eta}{\mu_{\alpha}^{1}-x_{k}}=g_{2}, \\
g l(0 \mid 2): \quad g_{1} \prod_{k=1}^{L} \frac{\mu_{\alpha}^{1}-x_{k}-\eta}{\mu_{\alpha}^{1}-x_{k}}=g_{2} \prod_{\gamma \neq \alpha}^{M_{2}} \frac{\mu_{\alpha}^{1}-\mu_{\gamma}^{1}-\eta}{\mu_{\alpha}^{1}-\mu_{\gamma}^{1}+\eta} .
\end{array}
$$

where $\alpha=1, \ldots, M_{2}$. Note that the equations for $g l(0 \mid 2)$ are obtained from those for $g l(2 \mid 0)$ by the transformation $\eta \rightarrow-\eta$. The spectrum is given by (5.3) with $\mathrm{p}(1)=0$ for $g l(2 \mid 0), g l(1 \mid 1)$ :

$$
H_{i}=g_{1} \prod_{k=1}^{L} \frac{x_{i}-x_{k}+\eta}{x_{i}-x_{k}} \prod_{\gamma=1}^{M_{2}} \frac{x_{i}-\mu_{\gamma}^{1}-\eta}{x_{i}-\mu_{\gamma}^{1}},
$$

and $\mathrm{p}(1)=1$ for $g l(0 \mid 2)$ which leads to the same expression with $\eta \rightarrow-\eta$.

\subsection{The QC correspondence: a direct proof}

Here we extend the result of [23] to supersymmetric spin chains.

Theorem 5.1 Substitute

$$
\dot{x}_{i}=-\eta H_{i}\left(\left\{x_{i}\right\}_{L},\left\{\mu_{\alpha}^{1}\right\}_{L_{1}}, g_{1}\right), \quad i=1, \ldots, L
$$

into the Lax matrix for the RS model (3.7), i.e. consider the matrix

$$
\left(\mathrm{Z}_{0}\right)_{i j}=\frac{\eta H_{i}}{x_{j}-x_{i}+\eta}
$$

(see (4.3)), where $H_{j}$ are eigenvalues (5.3) of the non-local Hamiltonians of the inhomogeneous graded $\operatorname{gl}(N \mid M)$ spin chain on $L$-sites with $N+M=K \leq L$ and the set $\left\{\mu_{\alpha}^{1}\right\}_{L_{1}}$ is 
taken from any solution $\left\{\mu_{\alpha}^{b}\right\}_{L_{b}}, b=1, \ldots, K-1$ of the Bethe equations (5.2). Then the spectrum of the Lax matrix (5.9) is of the form (4.4):

$$
\left.\operatorname{Spec} \mathbf{Z}_{0}\right|_{B E}=(\underbrace{g_{1}, \ldots, g_{1}}_{L-L_{1}}, \underbrace{g_{2}, \ldots, g_{2}}_{L_{1}-L_{2}}, \ldots, \underbrace{g_{K-1}, \ldots, g_{K-1}}_{L_{K-2}-L_{K-1}}, \underbrace{g_{K}, \ldots, g_{K}}_{L_{K-1}}) \text {. }
$$

Proof. The proof involves three steps. First, we recall the proof for the $g l(K \mid 0)$ case. Next, we show how to modify it for the $g l(0 \mid K)$ case. Lastly, the general case is processed by gluing together the previous two proofs in a proper way. When odd and even grading parameters are intermixed then one should apply the scheme of the proof given below each time the grading is changing.

Instead of $Z_{0}$ it is more convenient to deal with the transposed Lax matrix $Z_{0}^{t}$ given by

$$
\left(\mathrm{Z}_{0}^{\mathrm{t}}\right)_{i j}\left(\left\{\dot{x_{k}}\right\}_{L},\left\{x_{k}\right\}_{L}, \eta\right)=-\frac{\dot{x}_{j}}{x_{i}-x_{j}+\eta}=\frac{\eta H_{j}}{x_{i}-x_{j}+\eta}, \quad i, j=1, \ldots, L .
$$

Its spectrum coincides with that of $Z_{0}$.

1. $g l(K \mid 0)$ case. The proof given in [23] is based on the identity

$$
\operatorname{det}_{L \times L}\left(\mathcal{Z}\left(\left\{x_{i}\right\}_{L},\left\{y_{i}\right\}_{\tilde{L}}, g\right)-\lambda \mathbf{1}\right)=(g-\lambda)^{L-\tilde{L}} \operatorname{det}_{\tilde{L} \times \tilde{L}}\left(\widetilde{\mathcal{Z}}\left(\left\{y_{i}\right\}_{\tilde{L}},\left\{x_{i}\right\}_{L}, g\right)-\lambda \mathbf{1}\right)
$$

for the pair of $L \times L$ and $\tilde{L} \times \tilde{L}$ matrices

$$
\mathcal{Z}_{i j}\left(\left\{x_{k}\right\}_{L},\left\{y_{k}\right\}_{\tilde{L}}, g\right)=\frac{g \eta}{x_{i}-x_{j}+\eta} \prod_{k \neq j}^{L} \frac{x_{j}-x_{k}+\eta}{x_{j}-x_{k}} \prod_{\gamma=1}^{\tilde{L}} \frac{x_{j}-y_{\gamma}}{x_{j}-y_{\gamma}+\eta}
$$

and

$$
\widetilde{\mathcal{Z}}_{\alpha \beta}\left(\left\{y_{i}\right\}_{\tilde{L}},\left\{x_{i}\right\}_{L}, g\right)=\frac{g \eta}{y_{\alpha}-y_{\beta}+\eta} \prod_{\gamma \neq \beta}^{\tilde{L}} \frac{y_{\beta}-y_{\gamma}-\eta}{y_{\beta}-y_{\gamma}} \prod_{k=1}^{L} \frac{y_{\beta}-x_{k}}{y_{\beta}-x_{k}-\eta}
$$

(here $L \geq \tilde{L}$ ). In addition, we have

$$
\operatorname{det}_{L \times L}\left(\mathcal{Z}^{0}\left(\left\{x_{i}\right\}_{L}, g\right)-\lambda \mathbf{1}\right)=\operatorname{det}_{L \times L}\left(\widetilde{\mathcal{Z}}^{0}\left(\left\{y_{i}\right\}_{L}, g\right)-\lambda \mathbf{1}\right)=(g-\lambda)^{L},
$$

where

$$
\mathcal{Z}_{i j}^{0}\left(\left\{x_{k}\right\}_{L}, g\right)=\mathcal{Z}_{i j}\left(\left\{x_{k}\right\}_{L},\left\{y_{k}\right\}_{0}, g\right)=\frac{g \eta}{x_{i}-x_{j}+\eta} \prod_{k \neq j}^{L} \frac{x_{j}-x_{k}+\eta}{x_{j}-x_{k}}
$$

and

$$
\widetilde{\mathcal{Z}}_{\alpha \beta}^{0}\left(\left\{y_{i}\right\}_{L}, g\right)=\widetilde{\mathcal{Z}}_{\alpha \beta}\left(\left\{y_{i}\right\}_{L},\left\{x_{i}\right\}_{0}, g\right)=\frac{g \eta}{y_{\alpha}-y_{\beta}+\eta} \prod_{\gamma \neq \beta}^{L} \frac{y_{\beta}-y_{\gamma}-\eta}{y_{\beta}-y_{\gamma}} .
$$


The idea is to calculate $\operatorname{det}\left(Z_{0}-\lambda \mathbf{1}\right)$ by sequential usage of the identity (5.12) (which allows one to pass to a smaller matrix) and the Bethe equations (BE) (5.2) with $\mathrm{p}(b)=0$ for all b. Schematically, ${ }^{13}$ the procedure of the proof is as follows:

$$
\begin{aligned}
&\left\langle\mathrm{Z}_{0}^{\mathrm{t}}\right\rangle\left(-\eta\left\{H_{j}\right\}_{L},\left\{x_{j}\right\}_{L}, \eta\right) \stackrel{(5.3)}{=}\langle\mathcal{Z}\rangle\left(\left\{x_{i}-\eta\right\}_{L},\left\{\mu_{\alpha}^{1}\right\}_{L_{1}}, g_{1}\right) \stackrel{(5.12)}{\rightarrow} \\
&\langle\widetilde{\mathcal{Z}}\rangle\left(\left\{\mu_{\alpha}^{1}\right\}_{L_{1}},\left\{x_{i}-\eta\right\}_{L}, g_{1}\right) \stackrel{\mathrm{BE}_{b=1}}{=}\langle\mathcal{Z}\rangle\left(\left\{\mu_{\alpha}^{1}-\eta\right\}_{L_{1}},\left\{\mu_{\alpha}^{2}\right\}_{L_{2}}, g_{2}\right) \stackrel{(5.12)}{\rightarrow} \\
&\langle\widetilde{\mathcal{Z}}\rangle\left(\left\{\mu_{\alpha}^{2}\right\}_{L_{2}},\left\{\mu_{\alpha}^{1}-\eta\right\}_{L_{1}}, g_{2}\right) \stackrel{\mathrm{BE}_{b}=2}{=}\langle\mathcal{Z}\rangle\left(\left\{\mu_{\alpha}^{2}-\eta\right\}_{L_{2}},\left\{\mu_{\alpha}^{3}\right\}_{L_{3}}, g_{3}\right) \stackrel{(5.12)}{\rightarrow} \ldots
\end{aligned}
$$

Each time we use (5.12) the characteristic polynomial $\operatorname{det}(\mathcal{Z}-\lambda \mathbf{1})$ acquires the factor $\left(g_{b}-\lambda\right)^{L_{b-1}-L_{b}}$ except for the last step when we use (5.15) to get $\left(g_{K}-\lambda\right)^{L_{K-1}}$.

2. $g l(0 \mid K)$ case. It is easy to see that this case is similar to the previous one but the roles of $\mathcal{Z}$ and $\widetilde{\mathcal{Z}}$ in the scheme (5.18) get interchanged:

$$
\begin{gathered}
\left\langle\mathrm{Z}_{0}^{\mathrm{t}}\right\rangle\left(-\eta\left\{H_{j}\right\}_{L},\left\{x_{j}\right\}_{L}, \eta\right) \stackrel{(5.3)}{=}\langle\widetilde{\mathcal{Z}}\rangle\left(\left\{x_{i}+\eta\right\}_{L},\left\{\mu_{\alpha}^{1}\right\}_{L_{1}}, g_{1}\right) \stackrel{(5.12)}{\rightarrow} \\
\langle\mathcal{Z}\rangle\left(\left\{\mu_{\alpha}^{1}\right\}_{L_{1}},\left\{x_{i}+\eta\right\}_{L}, g_{1}\right) \stackrel{\mathrm{BE}_{b}=1}{=}\langle\widetilde{\mathcal{Z}}\rangle\left(\left\{\mu_{\alpha}^{1}+\eta\right\}_{L_{1}},\left\{\mu_{\alpha}^{2}\right\}_{L_{2}}, g_{2}\right) \stackrel{(5.12)}{\rightarrow} \\
\langle\mathcal{Z}\rangle\left(\left\{\mu_{\alpha}^{2}\right\}_{L_{2}},\left\{\mu_{\alpha}^{1}+\eta\right\}_{L_{1}}, g_{2}\right) \stackrel{\mathrm{BE}_{b}=2}{=}\langle\widetilde{\mathcal{Z}}\rangle\left(\left\{\mu_{\alpha}^{2}+\eta\right\}_{L_{2}},\left\{\mu_{\alpha}^{3}\right\}_{L_{3}}, g_{3}\right) \stackrel{(5.12)}{\rightarrow} \ldots
\end{gathered}
$$

Here we use the BE (5.2) with $\mathrm{p}(b)=1$ for all $b$.

3. $g l(N \mid M)$ case. We assume that $N, M \geq 1$. The scheme (5.18) works for $b=1, \ldots, N-1$ while (5.19) does for $b=N+1, \ldots, N+M$. In order to switch from the scheme (5.18) to (5.19) we need an intermediate step. It is accomplished by the BE (5.2) at $b=N$ :

$$
\mathrm{BE}_{b=N}: \quad g_{N} \prod_{\gamma=1}^{L_{N-1}} \frac{\mu_{\beta}^{N}-\mu_{\gamma}^{N-1}+\eta}{\mu_{\beta}^{N}-\mu_{\gamma}^{N-1}}=g_{N+1} \prod_{\gamma=1}^{L_{N+1}} \frac{\mu_{\beta}^{N}-\mu_{\gamma}^{N+1}+\eta}{\mu_{\beta}^{N}-\mu_{\gamma}^{N+1}} .
$$

Then for

$$
\begin{gathered}
\langle\widetilde{\mathcal{Z}}\rangle\left(\left\{\mu_{\alpha}^{N}\right\}_{L_{N}},\left\{\mu_{\alpha}^{N-1}-\eta\right\}_{L_{N-1}}, g_{N}\right) \\
=\frac{g_{N} \eta}{\mu_{\alpha}^{N}-\mu_{\beta}^{N}+\eta} \prod_{\gamma \neq \beta}^{L_{N}} \frac{\mu_{\beta}^{N}-\mu_{\gamma}^{N}-\eta}{\mu_{\beta}^{N}-\mu_{\gamma}^{N}} \prod_{\gamma=1}^{L_{N-1}} \frac{\mu_{\beta}^{N}-\mu_{\gamma}^{N-1}+\eta}{\mu_{\beta}^{N}-\mu_{\gamma}^{N-1}}
\end{gathered}
$$

we have:

$$
\langle\widetilde{\mathcal{Z}}\rangle\left(\left\{\mu_{\alpha}^{N}\right\}_{L_{N}},\left\{\mu_{\alpha}^{N-1}-\eta\right\}_{L_{N-1}}, g_{N}\right) \stackrel{(5.20)}{=}\langle\widetilde{\mathcal{Z}}\rangle\left(\left\{\mu_{\alpha}^{N}+\eta\right\}_{L_{N}},\left\{\mu_{\alpha}^{N+1}\right\}_{L_{N+1}}, g_{N+1}\right) .
$$

This finishes the proof.

\section{Concluding remarks}

Lastly, we would like to point out some unsolved problems and directions for further research.

\footnotetext{
${ }^{13}$ Here we symbolically write simply $\langle\mathcal{Z}\rangle$ for the characteristic polynomial $\operatorname{det}(\mathcal{Z}-\lambda \mathbf{1})$ with some overall factor.
} 
a) The properly taken limit $\eta \rightarrow 0$ should lead to the similar QC correspondence between graded quantum Gaudin models and classical Calogero-Moser systems. This is technically involved but rather straightforward procedure. The details will be published elsewhere.

b) A less straightforward but quite realistic program is the extension to the models based on trigonometric solutions to the (graded) Yang-Baxter equation (the graded XXZ magnets and corresponding vertex models of statistical mechanics). As the results of [8] suggest, one can expect the trigonometric RS model on the classical side of the QC correspondence. The precise relation between eigenvalues of the Lax matrix and the twist parameters of the spin chain is to be elaborated.

c) The extension to quantum integrable models with elliptic $R$-matrices is problematic. Conceivably this might require new ideas. At the same time, the most natural candidate for the classical part of the QC correspondence is the elliptic RS model. The role of the spectral parameter which enters its Lax matrix is to be clarified.

d) There is a well-known duality [24, 25] of the classical RS (and Calogero-Moser) type models when the action variables in a given system are treated as coordinates of particles in the dual one. Equivalently, the soliton-like tau-function whose zeros move as the RS particles becomes the spectral determinant for the dual system and vice versa. An interesting future perspective is to realize the meaning of this duality in the context of the quantun spin chains. Presumably, this duality implies some correspondence between spectra of different spin chains.

\section{A The higher T-operators through supercharacters}

Here we show how to derive (2.32). We have:

$$
\begin{aligned}
\mathbf{T}_{\lambda}(x)= & \operatorname{str}_{V_{\lambda}}\left[\left(\mathbf{I}+\frac{\eta}{x-x_{L}} \mathbf{P}_{\lambda}^{0 L}\right) \ldots\left(\mathbf{I}+\frac{\eta}{x-x_{2}} \mathbf{P}_{\lambda}^{02}\right)\left(\mathbf{I}+\frac{\eta}{x-x_{1}} \mathbf{P}_{\lambda}^{01}\right)\left(\pi_{\lambda}(\mathbf{g}) \otimes \mathbf{I}\right)\right] \\
= & \operatorname{str}_{V_{\lambda}} \pi_{\lambda}(\mathbf{g}) \mathbf{I}+\sum_{j} \frac{\eta}{x-x_{j}} \operatorname{str}_{V_{\lambda}}\left(\mathbf{P}_{\lambda}^{0 j}\left(\pi_{\lambda}(\mathbf{g}) \otimes \mathbf{I}\right)\right) \\
& +\sum_{i<j} \frac{\eta^{2}}{\left(x-x_{i}\right)\left(x-x_{j}\right)} \operatorname{str}_{V_{\lambda}}\left(\mathbf{P}_{\lambda}^{0 j} \mathbf{P}_{\lambda}^{0 i}\left(\pi_{\lambda}(\mathbf{g}) \otimes \mathbf{I}\right)\right) \\
& +\ldots+\frac{\eta^{L}}{\left(x-x_{1}\right) \ldots\left(x-x_{L}\right)} \operatorname{str}_{V_{\lambda}}\left(\mathbf{P}_{\lambda}^{0 L} \ldots \mathbf{P}_{\lambda}^{01}\left(\pi_{\lambda}(\mathbf{g}) \otimes \mathbf{I}\right)\right) .
\end{aligned}
$$


Plugging here the explicit form of $\mathbf{P}_{\lambda}^{0 j}(2.9)$, we get:

$$
\begin{aligned}
\mathbf{T}_{\lambda}(x)= & \operatorname{str}_{V_{\lambda}} \pi_{\lambda}(\mathbf{g}) \mathbf{I}+\sum_{j} \sum_{a b} \frac{\eta(-1)^{\mathbf{p}(b)} \mathbf{e}_{b a}^{(j)}}{x-x_{j}} \operatorname{str}_{V_{\lambda}}\left(\pi_{\lambda}\left(\mathbf{e}_{a b}\right) \pi_{\lambda}(\mathbf{g})\right) \\
& +\sum_{i<j} \sum_{a_{1}, b_{1} ; a_{2}, b_{2}} \frac{\eta^{2}(-1)^{\mathrm{p}\left(b_{1}\right)+\mathrm{p}\left(b_{2}\right)} \mathbf{e}_{b_{1} a_{1}}^{(i)} \mathbf{e}_{b_{2} a_{2}}^{(j)} \operatorname{str}_{V_{\lambda}}\left(\pi_{\lambda}\left(\mathbf{e}_{a_{2} b_{2}}\right) \pi_{\lambda}\left(\mathbf{e}_{a_{1} b_{1}}\right) \pi_{\lambda}(\mathbf{g})\right)}{\left(x-x_{1}\right)\left(x-x_{2}\right)} \\
& +\ldots \\
& +\sum_{\left\{a_{1}, b_{1} ; \ldots ; a_{L}, b_{L}\right\}} \frac{\eta^{L}(-1)^{\mathrm{p}\left(b_{1}\right)+\ldots+\mathrm{p}\left(b_{L}\right)} \mathbf{e}_{b_{1} a_{1}}^{(1)} \ldots \mathbf{e}_{b_{L} a_{L}}^{(L)}}{\left(x-x_{1}\right) \ldots\left(x-x_{L}\right)} \operatorname{str}_{V_{\lambda}}\left(\pi_{\lambda}\left(\mathbf{e}_{a_{L} b_{L}}\right) \ldots \pi_{\lambda}\left(\mathbf{e}_{a_{1} b_{1}}\right) \pi_{\lambda}(\mathbf{g})\right) .
\end{aligned}
$$

The last step is based on the following simple lemma:

Lemma A1. Let $\pi$ be a representation of $U(g l(N \mid M))$, $\chi$ its character and $\mathbf{h}_{1}, \mathbf{h}_{2}, \ldots, \mathbf{h}_{n}$ be any homogeneous elements of the superalgebra $g l(N \mid M)$ such that $\left[\mathbf{h}_{i}, \mathbf{h}_{i}\right]=0$ (here [, ] means the graded commutator). Then for any group element $\mathbf{g} \in G L(N \mid M)$ it holds:

$$
\operatorname{str}\left[\pi\left(\mathbf{h}_{1}\right) \pi\left(\mathbf{h}_{2}\right) \ldots \pi\left(\mathbf{h}_{n}\right) \pi(\mathbf{g})\right]=\left.\frac{\partial}{\partial \varepsilon_{n}} \ldots \frac{\partial}{\partial \varepsilon_{1}} \chi\left(e^{\varepsilon_{1} \mathbf{h}_{1}} \ldots e^{\varepsilon_{n} \mathbf{h}_{n}} \mathbf{g}\right)\right|_{\varepsilon_{i}=0},
$$

where it is implied that $\mathrm{p}\left(\varepsilon_{i}\right)=\mathrm{p}\left(\mathbf{h}_{i}\right)$.

The proof is simple. Since $\left[\mathbf{h}_{i}, \mathbf{h}_{i}\right]=0$, the exponents $e^{\varepsilon_{i} \mathbf{h}_{i}}$ are supergroup elements for any $\varepsilon_{i}$. Therefore, we have the chain of equalities

$$
\pi\left(e^{\varepsilon_{1} \mathbf{h}_{1}} \ldots e^{\varepsilon_{n} \mathbf{h}_{n}} \mathbf{g}\right)=\left[\prod_{j=1}^{\vec{n}} \pi\left(e^{\varepsilon_{j} \mathbf{h}_{j}}\right)\right] \pi(\mathbf{g})=\left[\prod_{j=1}^{\vec{n}} e^{\varepsilon_{j} \pi\left(\mathbf{h}_{j}\right)}\right] \pi(\mathbf{g}),
$$

from which it follows directly that

$$
\pi\left(\mathbf{h}_{1}\right) \pi\left(\mathbf{h}_{2}\right) \ldots \pi\left(\mathbf{h}_{n}\right) \pi(\mathbf{g})=\left.\frac{\partial}{\partial \varepsilon_{n}} \ldots \frac{\partial}{\partial \varepsilon_{1}} \pi\left(e^{\varepsilon_{1} \mathbf{h}_{1}} \ldots e^{\varepsilon_{n} \mathbf{h}_{n}} \mathbf{g}\right)\right|_{\varepsilon_{i}=0} .
$$

Taking supertrace of the both sides, we obtain (A.1). Applying the lemma to the case $\mathbf{h}_{i}=\mathbf{e}_{a_{i} b_{i}}$, we arrive at $(2.32)$.

\section{B Hamiltonian formulation of the RS model}

For completeness, we give here the Hamiltonian formulation of the $L$-particle RS model, including the higher flows. The Hamiltonian is

$$
\mathcal{H}_{1}=\sum_{i=1}^{L} e^{-\eta p_{i}} \prod_{k=1, \neq i}^{L} \frac{x_{i}-x_{k}+\eta}{x_{i}-x_{k}}
$$

with $\left\{p_{i}, x_{i}\right\}$ being the canonical variables with the standard Poisson brackets. The Hamiltonian equations of motion $\left(\begin{array}{c}\dot{x}_{i} \\ \dot{p}_{i}\end{array}\right)=\left(\begin{array}{c}\partial_{p_{i}} \mathcal{H}_{1} \\ -\partial_{x_{i}} \mathcal{H}_{1}\end{array}\right)$ give the connection between velocity and 
momentum

$$
\dot{x}_{i}=-\eta e^{-\eta p_{i}} \prod_{k=1, \neq i}^{L} \frac{x_{i}-x_{k}+\eta}{x_{i}-x_{k}}
$$

and the equations of motion (3.11).

The RS model is known to be integrable, with the higher integrals of motion in involution being given by $\mathcal{H}_{k}=\operatorname{tr} Z^{k}$, where $\mathbf{Z}$ is the Lax matrix of the model (3.7):

$$
\mathrm{Z}_{i j}=\frac{\dot{x}_{i}}{x_{i}-x_{j}-\eta}=\frac{\eta e^{-\eta p_{i}}}{x_{j}-x_{i}+\eta} \prod_{k=1, \neq i}^{L}\left(1+\frac{\eta}{x_{i}-x_{k}}\right)
$$

These integrals of motion can be regarded as Hamiltonians generating flows in the "higher times" $t_{k}$ via the Hamiltonian equations

$$
\left(\begin{array}{c}
\partial_{t_{k}} x_{i} \\
\partial_{t_{k}} p_{i}
\end{array}\right)=\left(\begin{array}{c}
\partial_{p_{i}} \mathcal{H}_{k} \\
-\partial_{i} \mathcal{H}_{k}
\end{array}\right), \quad k \geq 1
$$

Moreover, the dynamics in the higher time $t_{k}$ is precisely the one induced by the mKP flow on the roots of the tau function (3.1). The fact that the integrals of motion $\mathcal{H}_{k}$ are in involution agrees with the commutativity of the mKP flows. The proof is based on the linear problems (3.6), (3.14) and general relation (2.59). We will not repeat it here since it is technically involved. It can be found in $[9,19]$.

\section{Examples for small values of $L$ and limiting cases}

$\underline{L=1}$. For $L=1, \mathbf{T}_{\square}(x)=(\operatorname{str} \mathbf{g}) \mathbf{1}+\frac{\eta}{x-x_{1}} \sum_{a=1}^{K} g_{a} \mathbf{e}_{a a}$ and $\mathbf{H}_{1}=\sum_{a=1}^{K} g_{a} \mathbf{e}_{a a}=\mathbf{g}$. This case is trivial because there is only one Hamiltonian which is already diagonal. The system (4.8) is in a trivial agreement with this: it states that $H_{1}=g_{a}$. The master T-operator has the form

$$
\left.e^{-\operatorname{str} \xi(\mathbf{t}, \mathbf{g})} \frac{\mathrm{T}(x, \mathbf{t})}{\mathrm{T}_{\emptyset}(x)}\right|_{L=1}=\mathbf{I}+\eta \sum_{k \geq 1} \frac{k t_{k}\left(\mathbf{g}^{(1)}\right)^{k}}{x-x_{i}}
$$

$\underline{L=2}$. The case $L=2$ is more interesting. We have: $\mathbf{g}=g_{1} \mathbf{e}_{11}+g_{2} \mathbf{e}_{22}$,

$$
\begin{aligned}
\mathbf{T}_{\square}(x)=(\operatorname{str} \mathbf{g}) & \mathbf{1} \otimes \mathbf{1}+\frac{\eta}{x-x_{1}} \mathbf{g} \otimes \mathbf{1}+\frac{\eta}{x-x_{2}} \\
+ & \frac{\eta^{2}}{\left(x-x_{1}\right)\left(x-x_{2}\right)} \sum_{a, b=1}^{K}(-1)^{\mathrm{p}(a)} g_{b} \mathbf{e}_{b a} \otimes \mathbf{e}_{a b} .
\end{aligned}
$$

It is convenient to work in the basis $\mathbf{v}_{a} \otimes \mathbf{v}_{b}$ of the space $\mathcal{V}=\mathbb{C}^{N \mid M} \otimes \mathbb{C}^{N \mid M}$. The Hamiltonians act as follows:

$$
\begin{aligned}
& \mathbf{H}_{1} \mathbf{v}_{a} \otimes \mathbf{v}_{b}=g_{a} \mathbf{v}_{a} \otimes \mathbf{v}_{b}+\frac{(-1)^{\mathrm{p}(a) \mathrm{p}(b)} \eta g_{b}}{x_{1}-x_{2}} \mathbf{v}_{b} \otimes \mathbf{v}_{a} \\
& \mathbf{H}_{2} \mathbf{v}_{a} \otimes \mathbf{v}_{b}=g_{b} \mathbf{v}_{a} \otimes \mathbf{v}_{b}+\frac{(-1)^{\mathrm{p}(a) \mathrm{p}(b)} \eta g_{b}}{x_{2}-x_{1}} \mathbf{v}_{b} \otimes \mathbf{v}_{a}
\end{aligned}
$$


where we have used the rule

$$
\left(\mathbf{e}_{c d} \otimes \mathbf{e}_{d c}\right)\left(\mathbf{v}_{a} \otimes \mathbf{v}_{b}\right)=(-1)^{\mathrm{p}\left(\mathbf{e}_{d c}\right) \mathbf{p}\left(\mathbf{v}_{a}\right)} \mathbf{e}_{c d} \mathbf{v}_{a} \otimes \mathbf{e}_{d c} \mathbf{v}_{b}=(-1)^{(\mathbf{p}(d)+\mathrm{p}(c)) \mathrm{p}(a)} \delta_{d a} \delta_{c b} \mathbf{v}_{c} \otimes \mathbf{v}_{d} .
$$

Therefore, eigenvalues of $\mathbf{H}_{1}$ in the subspace of $\mathcal{V}$ spanned by the vectors $\mathbf{v}_{a_{1}} \otimes \mathbf{v}_{a_{2}}$ and $\mathbf{v}_{a_{2}} \otimes \mathbf{v}_{a_{1}}$ with some fixed $a_{1} \neq a_{2}$ are given by diagonalizing the $2 \times 2$ matrix

$$
\left(\begin{array}{cc}
g_{a_{1}} & \frac{(-1)^{\mathrm{p}\left(a_{1}\right) \mathrm{p}\left(a_{2}\right)} \eta g_{a_{1}}}{x_{1}-x_{2}} \\
\frac{(-1)^{\mathrm{p}\left(a_{1}\right) \mathrm{p}\left(a_{2}\right)} \eta g_{a_{2}}}{x_{1}-x_{2}} & g_{a_{2}}
\end{array}\right) .
$$

Then the spectrum of the operators $\left(\mathbf{H}_{1}, \mathbf{H}_{2}\right)$ is

$$
\begin{aligned}
\left(H_{1}, H_{2}\right)= & \left(g_{a_{1}}+\frac{(-1)^{\mathrm{p}\left(a_{1}\right)} \eta g_{a_{1}}}{x_{1}-x_{2}}, g_{a_{1}}+\frac{(-1)^{\mathrm{p}\left(a_{1}\right)} \eta g_{a_{1}}}{x_{2}-x_{1}}\right) \\
& \text { in } \mathbb{C v}_{a_{1}} \otimes \mathbf{v}_{a_{1}}\left(M_{a}=2 \delta_{a a_{1}}\right), \\
\left(H_{1}, H_{2}\right)= & \left(\frac{g_{a_{1}}+g_{a_{2}}+\sqrt{R}}{2}, \frac{g_{a_{1}}+g_{a_{2}}-\sqrt{R}}{2}\right),\left(\frac{g_{a_{1}}+g_{a_{2}}-\sqrt{R}}{2}, \frac{g_{a_{1}}+g_{a_{2}}+\sqrt{R}}{2}\right) \\
& \text { in } \mathbb{C v}_{a_{1}} \otimes \mathbf{v}_{a_{2}}+\mathbb{C v}_{a_{2}} \otimes \mathbf{v}_{a_{1}} \text { for } a_{1} \neq a_{2} \quad\left(M_{a}=\delta_{a a_{1}}+\delta_{a a_{2}}\right) .
\end{aligned}
$$

Here $R=\left(g_{a_{1}}-g_{a_{2}}\right)^{2}+\frac{4 \eta^{2} g_{a_{1}} g_{a_{2}}}{\left(x_{1}-x_{2}\right)^{2}}$ and $1 \leq a_{1}, a_{2} \leq K$ are some fixed indices.

Let us compare these results with solutions of the system (4.8) which in our case reduces to

$$
\left\{\begin{array}{l}
H_{1}+H_{2}=2 g_{a_{1}} \\
H_{1} H_{2}=g_{a_{1}}^{2}\left(1-\frac{\eta^{2}}{\left(x_{1}-x_{2}\right)^{2}}\right)
\end{array} \text { for } M_{a}=2 \delta_{a a_{1}}\right.
$$

and

$$
\left\{\begin{array}{l}
H_{1}+H_{2}=g_{a_{1}}+g_{a_{2}} \\
H_{1} H_{2}=g_{a_{1}} g_{a_{2}}\left(1-\frac{\eta^{2}}{\left(x_{1}-x_{2}\right)^{2}}\right)
\end{array} \text { for } M_{a}=\delta_{a a_{1}}+\delta_{a a_{2}} .\right.
$$

Each system has two solutions for the pair $\left(H_{1}, H_{2}\right)$. One can easily check that the two solutions of the former system are just (C.5) for the two possible values $\mathrm{p}\left(a_{1}\right)=0,1$ while the two solutions of the latter one are given by (C.6).

The master T-operator has the form

$$
\begin{aligned}
& \left.e^{-\operatorname{str} \xi(\mathbf{t}, \mathbf{g})} \frac{\mathbf{T}(x, \mathbf{t})}{\mathbf{T}_{\emptyset}(x)}\right|_{L=2}=\mathbf{I}+\eta \sum_{i=1}^{2} \sum_{k \geq 1} \frac{k t_{k}\left(\mathbf{g}^{(i)}\right)^{k}}{x-x_{i}}+\frac{\eta^{2}}{\left(x-x_{1}\right)\left(x-x_{2}\right)} \\
& \times\left[\left(\sum_{k_{1} \geq 1} k_{1} t_{k_{1}}\left(\mathbf{g}^{(1)}\right)^{k_{1}}\right)\left(\sum_{k_{2} \geq 1} k_{2} t_{k_{2}}\left(\mathbf{g}^{(2)}\right)^{k_{2}}\right)+\sum_{k \geq 1} k t_{k} \sum_{\alpha=0}^{k-1} \mathbf{P}_{12}\left(\mathbf{g}^{(1)}\right)^{\alpha}\left(\mathbf{g}^{(2)}\right)^{k-\alpha}\right] .
\end{aligned}
$$

$\underline{L=3}$. We start with the explicit form of the Hamiltonians $\mathbf{H}_{i}$. Let us introduce the short-hand notation $\mathrm{p}_{i}:=(-1)^{\mathrm{p}\left(a_{i}\right)}, \mathrm{p}_{i j}:=(-1)^{\mathrm{p}\left(a_{i}\right) \mathrm{p}\left(a_{j}\right)}$. In the 3-dimensional subspace 
spanned by the vectors $\mathbf{v}_{a_{1}} \otimes \mathbf{v}_{a_{1}} \otimes \mathbf{v}_{a_{2}}, \mathbf{v}_{a_{1}} \otimes \mathbf{v}_{a_{2}} \otimes \mathbf{v}_{a_{1}}$ and $\mathbf{v}_{a_{2}} \otimes \mathbf{v}_{a_{1}} \otimes \mathbf{v}_{a_{1}}$ for $a_{1} \neq a_{2}$, we have

$$
\begin{aligned}
& \mathbf{H}_{1}=\left(\begin{array}{ccc}
g_{a_{1}}\left(\frac{\eta \mathrm{p}_{1}}{x_{12}}+1\right) & \frac{\eta^{2} g_{a_{1}} \mathrm{p}_{1} \mathrm{p}_{12}}{x_{12} x_{13}} & \frac{\eta g_{a_{1}} \mathrm{p}_{1}}{x_{13}} \\
0 & g_{a_{1}}\left(\frac{\eta \mathrm{p}_{1}}{x_{13}}+1\right) & \frac{\eta g_{a_{1}} \mathrm{p}_{12}\left(x_{13}+\eta \mathrm{p}_{1}\right)}{x_{12} x_{13}} \\
\frac{\eta g_{a_{2}}\left(x_{12} \mathrm{p}_{1}+\eta\right)}{x_{12} x_{13}} & \frac{\eta g_{a_{2}} \mathrm{p}_{12}}{x_{12}} & g_{a_{2}}
\end{array}\right), \\
& \mathbf{H}_{2}=\left(\begin{array}{ccc}
g_{a_{1}}\left(-\frac{\eta \mathrm{p}_{1}}{x_{12}}+1\right) & \frac{\eta g_{a_{1}} \mathrm{p}_{12}\left(x_{12}-\eta \mathrm{p}_{1}\right)}{x_{12} x_{23}} & 0 \\
\frac{\eta g_{a_{2}} \mathrm{p}_{12}}{x_{23}} & g_{a_{2}} & -\frac{\eta g_{a_{1}} \mathrm{p}_{12}\left(x_{23}+\eta \mathrm{p}_{1}\right)}{x_{12} x_{23}} \\
\frac{-\eta^{2} g_{a_{2}}}{x_{12} x_{23}} & -\frac{\eta g_{a_{2}} \mathrm{p}_{12}}{x_{12}} & g_{a_{1}}\left(\frac{\eta \mathrm{p}_{1}}{x_{23}}+1\right)
\end{array}\right), \\
& \mathbf{H}_{3}=\left(\begin{array}{ccc}
g_{a_{2}} & -\frac{\eta g_{a_{1}} \mathrm{p}_{12}\left(x_{13}-\eta \mathrm{p}_{1}\right)}{x_{23} x_{13}} & -\frac{\eta g_{a_{1}} \mathrm{p}_{1}}{x_{13}} \\
-\frac{\eta g_{a_{2}} \mathrm{p}_{12}}{x_{23}} & g_{a_{1}}\left(-\frac{\eta \mathrm{p}_{1}}{x_{13}}+1\right) & \frac{\eta^{2} g_{a_{1}} \mathrm{p}_{1} \mathrm{p}_{12}}{x_{13} x_{23}} \\
-\frac{\eta g_{a_{2}}\left(x_{23} \mathrm{p}_{1}-\eta\right)}{x_{13} x_{23}} & 0 & g_{a_{1}}\left(-\frac{\eta \mathrm{p}_{1}}{x_{23}}+1\right)
\end{array}\right) .
\end{aligned}
$$

In the 6-dimensional subspace spanned by the vectors $\left\{\mathbf{v}_{a_{\tau(1)}} \otimes \mathbf{v}_{a_{\tau(2)}} \otimes \mathbf{v}_{a_{\tau(3)}}\right\}_{\tau \in S_{3}}$, where $S_{3}$ is the permutation group over $\{1,2,3\}$ and $a_{i} \neq a_{j}$ if $i \neq j$, we have

$$
\mathbf{H}_{1}=\left(\begin{array}{cccccc}
g_{a_{1}} & 0 & \frac{\eta g_{a_{1}} \mathrm{p}_{12}}{x_{12}} & \frac{\eta^{2} g_{a_{1}} \mathrm{p}_{12} \mathrm{p}_{13}}{x_{12} x_{13}} & 0 & \frac{\eta g_{a_{1}} \mathrm{p}_{12} \mathrm{p}_{13} \mathrm{p}_{23}}{x_{13}} \\
0 & g_{a_{1}} & 0 & \frac{\eta g_{a_{1}} \mathrm{p}_{12} \mathrm{p}_{13} \mathrm{p}_{23}}{x_{13}} & \frac{\eta g_{a_{1}} \mathrm{p}_{13}}{x_{12}} & \frac{\eta^{2} g_{a_{1}} \mathrm{p}_{12} \mathrm{p}_{13}}{x_{12} x_{13}} \\
\frac{\eta g_{a_{2}} \mathrm{p}_{12}}{x_{12}} & \frac{\eta^{2} g_{a_{2}} \mathrm{p}_{12} \mathrm{p}_{23}}{x_{12} x_{13}} & g_{a_{2}} & 0 & \frac{\eta g_{a_{2}} \mathrm{p}_{12} \mathrm{p}_{13} \mathrm{p}_{23}}{x_{13}} & 0 \\
0 & \frac{\eta g_{a_{2}} \mathrm{p}_{12} \mathrm{p}_{13} \mathrm{p}_{23}}{x_{13}} & 0 & g_{a_{2}} & \frac{\eta^{2} g_{a_{2}} \mathrm{p}_{12} \mathrm{p}_{23}}{x_{12} x_{13}} & \frac{\eta g_{a_{2}} \mathrm{p}_{23}}{x_{12}} \\
\frac{\eta^{2} g_{a_{3}} \mathrm{p}_{13} \mathrm{p}_{23}}{x_{12} x_{13}} & \frac{\eta g_{a_{3}} \mathrm{p}_{13}}{x_{12}} & \frac{\eta g_{a_{3}} \mathrm{p}_{12} \mathrm{p}_{13} \mathrm{p}_{23}}{x_{13}} & 0 & g_{a_{3}} & 0 \\
\frac{\eta g_{a_{3}} \mathrm{p}_{12} \mathrm{p}_{13} \mathrm{p}_{23}}{x_{13}} & 0 & \frac{\eta^{2} g_{a_{3}} \mathrm{p}_{13} \mathrm{p}_{23}}{x_{12} x_{13}} & \frac{\eta g_{a_{3}} \mathrm{p}_{23}}{x_{12}} & 0 & g_{a_{3}}
\end{array}\right),
$$

$$
\mathbf{H}_{2}=\left(\begin{array}{cccccc}
g_{a_{2}} & \frac{\eta g_{a_{2}} \mathrm{p}_{23}}{x_{23}} & \frac{\eta g_{a_{1}} \mathrm{p}_{12}}{x_{21}} & -\frac{\eta^{2} g_{a_{1}} \mathrm{p}_{12} \mathrm{p}_{13}}{x_{12} x_{23}} & 0 & 0 \\
\frac{\eta g_{a_{3}} \mathrm{p}_{23}}{x_{23}} & g_{a_{3}} & 0 & 0 & \frac{\eta g_{a_{1}} \mathrm{p}_{13}}{x_{21}} & -\frac{\eta^{2} g_{a_{1}} \mathrm{p}_{12} \mathrm{p}_{13}}{x_{12} x_{23}} \\
\frac{\eta g_{a_{2}} \mathrm{p}_{12}}{x_{21}} & -\frac{\eta^{2} g_{a_{2}} \mathrm{p}_{12} \mathrm{p}_{23}}{x_{12} x_{23}} & g_{a_{1}} & \frac{\eta g_{a_{1}} \mathrm{p}_{13}}{x_{23}} & 0 & 0 \\
0 & 0 & \frac{\eta g_{a_{3}} \mathrm{p}_{13}}{x_{23}} & g_{a_{3}} & -\frac{\eta^{2} g_{a_{2}} \mathrm{p}_{12} \mathrm{p}_{23}}{x_{12} x_{23}} & \frac{\eta g_{a_{2}} \mathrm{p}_{23}}{x_{21}} \\
-\frac{\eta^{2} g_{a_{3}} \mathrm{p}_{13} \mathrm{p}_{23}}{x_{12} x_{23}} & \frac{\eta g_{a_{3}} \mathrm{p}_{13}}{x_{21}} & 0 & 0 & g_{a_{1}} & \frac{\eta g_{a_{1}} \mathrm{p}_{12}}{x_{23}} \\
0 & 0 & -\frac{\eta^{2} g_{a_{3}} \mathrm{p}_{13} \mathrm{p}_{23}}{x_{12} x_{23}} & \frac{\eta g_{a_{3}} \mathrm{p}_{23}}{x_{21}} & \frac{\eta g_{a_{2}} \mathrm{p}_{12}}{x_{23}} & g_{a_{2}}
\end{array}\right),
$$




$$
\mathbf{H}_{3}=\left(\begin{array}{cccccc}
g_{a_{3}} & \frac{\eta g_{a_{2}} \mathrm{p}_{23}}{x_{32}} & 0 & -\frac{\eta^{2} g_{a_{1}} \mathrm{p}_{12} \mathrm{p}_{13}}{x_{13} x_{32}} & 0 & \frac{\eta g_{a_{1}} \mathrm{p}_{12} \mathrm{p}_{13} \mathrm{p}_{23}}{x_{31}} \\
\frac{\eta g_{a_{3}} \mathrm{p}_{23}}{x_{32}} & g_{a_{2}} & 0 & \frac{\eta g_{a_{1}} \mathrm{p}_{12} \mathrm{p}_{13} \mathrm{p}_{23}}{x_{31}} & 0 & -\frac{\eta^{2} g_{a_{1}} \mathrm{p}_{12} \mathrm{p}_{13}}{x_{13} x_{32}} \\
0 & -\frac{\eta^{2} g_{a_{2}} \mathrm{p}_{12} \mathrm{p}_{23}}{x_{13} x_{32}} & g_{a_{3}} & \frac{\eta g_{a_{1}} \mathrm{p}_{13}}{x_{32}} & \frac{\eta g_{a_{2}} \mathrm{p}_{12} \mathrm{p}_{13} \mathrm{p}_{23}}{x_{31}} & 0 \\
0 & \frac{\eta g_{a_{2}} \mathrm{p}_{12} \mathrm{p}_{13} \mathrm{p}_{23}}{x_{31}} & \frac{\eta g_{a_{3}} \mathrm{p}_{13}}{x_{32}} & g_{a_{1}} & -\frac{\eta^{2} g_{a_{2}} \mathrm{p}_{12} \mathrm{p}_{23}}{x_{13} x_{32}} & 0 \\
-\frac{\eta g_{a_{3}}}{x_{13} x_{32}} & 0 & \frac{\eta g_{a_{3}} \mathrm{p}_{12} \mathrm{p}_{13} \mathrm{p}_{23}}{x_{31}} & 0 & g_{a_{2}} & \frac{\eta g_{a_{1}} \mathrm{p}_{12}}{x_{32}} \\
\frac{\eta g_{a_{3}} \mathrm{p}_{12} \mathrm{p}_{13} \mathrm{p}_{23}}{x_{31}} & 0 & -\frac{\eta^{2} g_{a_{3}} \mathrm{p}_{13} \mathrm{p}_{23}}{x_{13} x_{32}} & 0 & \frac{\eta g_{a_{2}} \mathrm{p}_{12}}{x_{32}} & g_{a_{1}}
\end{array}\right) .
$$

For general $g_{1}, g_{2}, g_{3}$ the analytic diagonalization of these matrices is not possible. However, it can be done numerically for any particular values of the parameters.

For $L=3$ the system (4.8) looks as follows:

$$
\left\{\begin{array}{l}
H_{1}+H_{2}+H_{3}=C_{1}\left(\left\{M_{a}\right\}\right) \\
\frac{H_{1} H_{2}}{1-\frac{\eta^{2}}{x_{12}^{2}}}+\frac{H_{2} H_{3}}{1-\frac{\eta^{2}}{x_{23}^{2}}}+\frac{H_{1} H_{3}}{1-\frac{\eta^{2}}{x_{13}^{2}}}=C_{2}\left(\left\{M_{a}\right\}\right) \\
\frac{H_{1} H_{2} H_{3}}{\left(1-\frac{\eta^{2}}{x_{12}^{2}}\right)\left(1-\frac{\eta^{2}}{x_{23}^{2}}\right)\left(1-\frac{\eta^{2}}{x_{13}^{2}}\right)}=C_{3}\left(\left\{M_{a}\right\}\right)
\end{array}\right.
$$

where $x_{i j} \equiv x_{i}-x_{j}$,

$$
\begin{aligned}
& C_{1}\left(\left\{M_{a}\right\}\right)=\sum_{a=1}^{K} M_{a} g_{a} \\
& C_{2}\left(\left\{M_{a}\right\}\right)=\frac{1}{2}\left(\sum_{a=1}^{K} M_{a} g_{a}\right)^{2}-\frac{1}{2} \sum_{a=1}^{K} M_{a} g_{a}^{2} \\
& C_{3}\left(\left\{M_{a}\right\}\right)=\frac{1}{6}\left(\sum_{a=1}^{K} M_{a} g_{a}\right)^{3}-\frac{1}{2}\left(\sum_{a=1}^{K} M_{a} g_{a}^{2}\right)\left(\sum_{b=1}^{K} M_{b} g_{b}\right)+\frac{1}{3} \sum_{a=1}^{K} M_{a} g_{a}^{3} .
\end{aligned}
$$

and $M_{1}+\ldots+M_{K}=3$. Correspondingly, there are 3 possibilities:

a) $M_{a}=3 \delta_{a a_{1}}$, then $C_{1}=3 g_{a_{1}}, C_{2}=3 g_{a_{1}}^{2}, C_{3}=g_{a_{1}}^{3}$;

b) $M_{a}=2 \delta_{a a_{1}}+\delta_{a a_{2}}\left(a_{1} \neq a_{2}\right)$, then $C_{1}=2 g_{a_{1}}+g_{a_{2}}, C_{2}=2 g_{a_{1}} g_{a_{2}}+g_{a_{1}}^{2}, C_{3}=g_{a_{1}}^{2} g_{a_{2}}$;

c) $M_{a}=\delta_{a a_{1}}+\delta_{a a_{2}}+\delta_{a a_{3}}\left(a_{0} \neq a_{2} \neq a_{3}\right)$, then $C_{1}=g_{a_{1}}+g_{a_{2}}+g_{a_{3}}, C_{2}=g_{a_{1}} g_{a_{2}}+g_{a_{1}} g_{a_{3}}+g_{a_{2}} g_{a_{3}}, C_{3}=g_{a_{1}} g_{a_{2}} g_{a_{3}}$.

Here $1 \leq a_{1}, a_{2}, a_{3} \leq K$ are 3 fixed indices.

It is instructive to solve the system and to figure out how the solutions correspond to the spectra of the spin chains. In the case a) we have found the following sets of solutions 
for the triple $\left(H_{1}, H_{2}, H_{3}\right)$ :

$$
\begin{aligned}
& H_{j}=g_{a_{1}} \prod_{k=1, \neq j}^{3}\left(1 \pm \frac{\eta}{x_{j k}}\right), \quad j=1,2,3, \\
& H_{\alpha}=g_{a_{1}}\left(1+\frac{\eta^{2} \pm \eta \sqrt{Q}}{2 x_{\alpha \beta} x_{\gamma \alpha}}\right), \quad Q \equiv 2\left(x_{12}^{2}+x_{13}^{2}+x_{23}^{2}\right)-3 \eta^{2},
\end{aligned}
$$

where $\{\alpha, \beta, \gamma\}$ in the second line stands for any cyclic permutation of $\{1,2,3\}$. The multiplicity of each of the solutions (C.15) is 1 and that of (C.16) is 2 (thus there are $2 \times 1+2 \times 2=6$ solutions in total). For the case b) with $g_{a_{1}}=g_{a_{2}}$ or for the case c) with $g_{a_{1}}=g_{a_{2}}=g_{a_{3}}$ the system of equations has exactly the same form as for the case a). Therefore, these three cases share the same sets of solutions.

Let us give a more detailed description based on the analytic solutions as well as on numerical calculations. By a solution of (C.14) we mean here the ordered set $\left(H_{1}, H_{2}, H_{3}\right)$.

In case a), $\left(M_{1}, M_{2}, M_{3}\right)=(3,0,0)$, there are 6 solutions. One of them (the one in (C.15) with plus) coincides with the eigenvalues of $\left(\mathbf{H}_{1}, \mathbf{H}_{2}, \mathbf{H}_{3}\right)$ in the one-dimensional space $\mathcal{V}(3,0,0)$ with the grading parameter $\mathrm{p}(1)=0$. Another one (the one in (C.15) with minus) coincides with the eigenvalue in the space $\mathcal{V}(3,0,0)$ but with the grading parameter $\mathrm{p}(1)=1$ (that is the same as changing $\eta \rightarrow-\eta$ ). The rest 4 solutions (C.16) do not correspond to eigenvalues of $\left(\mathbf{H}_{1}, \mathbf{H}_{2}, \mathbf{H}_{3}\right)$ in the same model if all the twist parameters are different. Instead, they can be found among the sets of eigenvalues in the 3-dimensional space $\mathcal{V}(2,1,0)$ for the model with $g_{1}=g_{2}$ and $\mathrm{p}(1)=0,1$ or in the 6 -dimensional space $\mathcal{V}(1,1,1)$ for the model with $g_{1}=g_{2}=g_{3}$ independently of the grading parameters.

In case b), $\left(M_{1}, M_{2}, M_{3}\right)=(2,1,0)$, among 6 solutions of (C.14), three coincide with three sets of eigenvalues of $\left(\mathbf{H}_{1}, \mathbf{H}_{2}, \mathbf{H}_{3}\right)$ in the 3 -dimensional space $\mathcal{V}(2,1,0)$ with the grading parameter $p(1)=0$. The rest three solutions coincide with three sets of eigenvalues in the space $\mathcal{V}(2,1,0)$ but with the grading parameter $\mathrm{p}(1)=1$.

Lastly, in case c), $\left(M_{1}, M_{2}, M_{3}\right)=(1,1,1)$, the 6 solutions of (C.14) coincide with the 6 sets of eigenvalues of $\left(\mathbf{H}_{1}, \mathbf{H}_{2}, \mathbf{H}_{3}\right)$ in the six-dimensional space $\mathcal{V}(1,1,1)$. They do not depend on the grading parameters. It is the case of "general position". The other cases can be obtained form it by a degeneration procedure. We conjecture that this is true for any $L$.

The master T-operator for $L=3$ has the form

$$
\begin{aligned}
& \left.e^{-\operatorname{str} \xi(\mathbf{t}, \mathbf{g})} \frac{\mathbf{T}(x, \mathbf{t})}{\mathbf{T}_{\emptyset}(x)}\right|_{L=3}=\mathbf{I}+\eta \sum_{i=1}^{3} \sum_{k \geq 1} \frac{k t_{k}\left(\mathbf{g}^{(i)}\right)^{k}}{x-x_{i}}+\eta^{2} \sum_{1 \leq i_{1}<i_{2} \leq 3} \frac{1}{\left(x-x_{i_{1}}\right)\left(x-x_{i_{2}}\right)} \\
& \times\left(\left(\sum_{k_{1} \geq 1} k_{1} t_{k_{1}}\left(\mathbf{g}^{\left(i_{1}\right)}\right)^{k_{1}}\right)\left(\sum_{k_{2} \geq 1} k_{2} t_{k_{2}}\left(\mathbf{g}^{\left(i_{2}\right)}\right)^{k_{2}}\right)+\sum_{k \geq 1} k t_{k} \sum_{\alpha=0}^{k-1} \mathbf{P}_{i_{1} i_{2}}\left(\mathbf{g}^{\left(i_{1}\right)}\right)^{\alpha}\left(\mathbf{g}^{\left(i_{2}\right)}\right)^{k-\alpha}\right) \\
& +\frac{\eta^{3}}{\left(x-x_{1}\right)\left(x-x_{2}\right)\left(x-x_{3}\right)}[ \\
& \left(\sum_{k_{1} \geq 1} k_{1} t_{k_{1}}\left(\mathbf{g}^{(1)}\right)^{k_{1}}\right)\left(\sum_{k_{2} \geq 1} k_{2} t_{k_{2}}\left(\mathbf{g}^{(2)}\right)^{k_{2}}\right)\left(\sum_{k_{3} \geq 1} k_{3} t_{k_{3}}\left(\mathbf{g}^{(3)}\right)^{k_{3}}\right)
\end{aligned}
$$




$$
\begin{aligned}
& +\left(\sum_{k_{1} \geq 1} k_{1} t_{k_{1}}\left(\mathbf{g}^{(1)}\right)^{k_{1}}\right)\left(\sum_{k \geq 1} k t_{k} \sum_{\alpha=0}^{k-1} \mathbf{P}_{23}\left(\mathbf{g}^{(2)}\right)^{\alpha}\left(\mathbf{g}^{(3)}\right)^{k-\alpha}\right) \\
& +\left(\sum_{k_{2} \geq 1} k_{2} t_{k_{2}}\left(\mathbf{g}^{(2)}\right)^{k_{2}}\right)\left(\sum_{k \geq 1} k t_{k} \sum_{\alpha=0}^{k-1} \mathbf{P}_{13}\left(\mathbf{g}^{(1)}\right)^{\alpha}\left(\mathbf{g}^{(3)}\right)^{k-\alpha}\right) \\
& +\left(\sum_{k_{3} \geq 1} k_{3} t_{k_{3}}\left(\mathbf{g}^{(3)}\right)^{k_{3}}\right)\left(\sum_{k \geq 1} k t_{k} \sum_{\alpha=0}^{k-1} \mathbf{P}_{12}\left(\mathbf{g}^{(1)}\right)^{\alpha}\left(\mathbf{g}^{(2)}\right)^{k-\alpha}\right) \\
& +\sum_{k \geq 1} k t_{k} \sum_{\alpha_{2}=0}^{k-1} \mathbf{P}_{23}\left(\sum_{\alpha_{1}=0}^{\alpha_{2}-1} \mathbf{P}_{12}\left(\mathbf{g}^{(1)}\right)^{\alpha_{1}}\left(\mathbf{g}^{(2)}\right)^{\alpha_{2}-\alpha_{1}}\right)\left(\mathbf{g}^{(3)}\right)^{k-\alpha_{2}} \\
& \left.+\sum_{k \geq 1} k t_{k} \sum_{\alpha_{2}=0}^{k-1} \mathbf{P}_{23}\left(\mathbf{g}^{(2)}\right)^{\alpha_{2}}\left(\sum_{\alpha_{1}=0}^{k-\alpha_{2}-1} \mathbf{P}_{13}\left(\mathbf{g}^{(1)}\right)^{\alpha_{1}}\left(\mathbf{g}^{(3)}\right)^{k-\alpha_{1}-\alpha_{2}}\right)\right] .
\end{aligned}
$$

Limiting cases. There are two limits, where one can obtain the spectrum explicitly: $\eta \rightarrow$ $0, \infty$. Here we assume that the twist parameters do not depend on $\eta$. Thus, the limit $\eta \rightarrow 0$ discussed here differs from the one to the Gaudin model.

(i) $\eta \rightarrow 0$. The operator $\mathbf{H}_{j}^{(0)}=\lim _{\eta \rightarrow 0} \mathbf{H}_{j}=\mathbf{g}^{(j)}$ is diagonal in the basis $\mathbf{v}_{a_{1}} \otimes \mathbf{v}_{a_{2}} \otimes$ $\ldots \otimes \mathbf{v}_{a_{L}}$. It has the eigenvalues $g_{a_{j}}$. The degeneracy of each eigenvalue is $K^{L-1}$. The system of algebraic equations (4.8) degenerates into (4.11). The solutions of (4.11) correspond to this case if $L \leq K$ and all the $\left\{a_{i}\right\}_{i=1}^{L}$ are distinct. If some of $M_{i}$ 's are bigger than 1 (this is always the case if $L>K$ ), then the eigenvalues of the Hamiltonians can be obtained by merging some of the twist parameters in (4.11). Such solutions of (4.11) also correspond to the above eigenvalues when some of the $a_{i}^{\prime} s$ or $g_{k}$ 's coincide.

(ii) $\eta \rightarrow \infty$. Consider the operator $\mathbf{H}_{j}^{(\infty)}=\lim _{\eta \rightarrow \infty} \eta^{1-L} \mathbf{H}_{j}$. It acts on the basis vectors as follows (see (2.15)):

$$
\mathbf{H}_{j}^{(\infty)}\left(\mathbf{v}_{a_{1}} \otimes \mathbf{v}_{a_{2}} \otimes \ldots \otimes \mathbf{v}_{a_{L}}\right)=\frac{(-1)^{\mathrm{p}\left(a_{L}\right) \sum_{k=1}^{L-1} \mathrm{p}\left(a_{k}\right)} g_{a_{L}}}{\prod_{k=1, \neq j}^{L}\left(x_{j}-x_{k}\right)}\left(\mathbf{v}_{a_{L}} \otimes \mathbf{v}_{a_{1}} \otimes \ldots \otimes \mathbf{v}_{a_{L-1}}\right) .
$$

Then we find that $\mathbf{H}_{j}^{(\infty)}$ has an eigenvector

$$
\sum_{k=0}^{L-1}(-1)^{-\frac{2 \alpha k}{L}+\sum_{i=0}^{k-1} \mathrm{p}\left(a_{\sigma^{i}(L)}\right) \sum_{l=1}^{L-1} \mathrm{p}\left(a_{\sigma^{i}(l)}\right)} \prod_{i=1}^{L} g_{a_{\sigma^{k}(i)}^{\frac{L-i}{L}}}\left(\mathbf{v}_{a_{\sigma^{k}(1)}} \otimes \ldots \otimes \mathbf{v}_{a_{\sigma^{k}(L)}}\right)
$$

for each $\alpha \in\{0,1, \ldots, L-1\}$, where $\sigma(i)=i-1$ for $i \in\{2,3, \ldots, L\}, \sigma(1)=L$. The corresponding eigenvalue is

$$
\frac{(-1)^{\frac{2 \alpha}{L}} \prod_{k=1}^{L} g_{a_{k}}^{\frac{1}{L}}}{\prod_{k=1, \neq j}^{L}\left(x_{j}-x_{k}\right)}
$$


Apparently, degeneracy of each eigenvalue is $(L-1)$ ! if $a_{i} \neq a_{k}$ for any $i \neq k$. Note that the eigenvalue (C.20) does not depend on the grading parameters, although the corresponding eigenvector (C.19) does.

The system (4.8) in the limit $\eta \rightarrow \infty$ reduces to

$$
\sum_{1 \leq i_{1}<\ldots<i_{n} \leq L} H_{i_{1}}^{(\infty)} \ldots H_{i_{n}}^{(\infty)} \prod_{\alpha=1}^{n} \prod_{\beta=1, \neq \alpha}^{n}\left(x_{i_{\alpha}}-x_{i_{\beta}}\right)=\delta_{n, L} C_{L}, \quad n=1, \ldots, L,
$$

where we denote $H_{j}^{(\infty)}=\lim _{\eta \rightarrow \infty} \eta^{1-L} H_{j}$. Note that (C.20) satisfies (C.21), at least for the case $L=K$ and $M_{j}=1$ (for all $1 \leq j \leq K$ ). This fact follows from the following identities:

$$
\sum_{\substack{I \subset\{1,2, \ldots, L\},|I|=n, \bar{I}=\{1,2, \ldots, L\} \backslash I}} \frac{1}{\prod_{\alpha \in I} \prod_{\beta \in \bar{I}}\left(x_{\alpha}-x_{\beta}\right)}=0, \quad n=1,2, \ldots, L-1 .
$$

Here we assume that all the $\left\{x_{i}\right\}_{i=1}^{L}$ are distinct. The case $L=2$ is trivial. Let us take $L \geq 3$. One can regard the left hand side of (C.22) as a function of $x_{1}$ and denote it as $f\left(x_{1}\right)$. Apparently, $f\left(x_{1}\right)$ has a simple pole at $x_{1}=x_{i}$ for each $i \in\{2,3, \ldots, L\}$. However, the residues of $f\left(x_{1}\right)$ at all these poles vanish. For example, the terms that can contribute to the residue at $x_{1}=x_{2}$ (coming from the terms with $1 \in I, 2 \in \bar{I}$ or $2 \in I, 1 \in \bar{I}$ ) have the form

$$
\begin{gathered}
\sum_{\substack{J \subset\{3,4, \ldots, L\},|J|=n-2, \bar{J}=\{3,4, \ldots, L\} \backslash J}} \frac{1}{x_{1}-x_{2}}\left\{\frac{1}{\prod_{\beta \in \bar{J}}\left(x_{1}-x_{\beta}\right) \prod_{\alpha \in J}\left(x_{\alpha}-x_{2}\right) \prod_{\alpha \in J} \prod_{\beta \in \bar{J}}\left(x_{\alpha}-x_{\beta}\right)}\right. \\
\left.-\frac{1}{\prod_{\beta \in \bar{J}}\left(x_{2}-x_{\beta}\right) \prod_{\alpha \in J}\left(x_{\alpha}-x_{1}\right) \prod_{\alpha \in J} \prod_{\beta \in \bar{J}}\left(x_{\alpha}-x_{\beta}\right)}\right\} .
\end{gathered}
$$

One can easily see that the residue at $x_{1}=x_{2}$ is 0 . All other cases are considered in a similar way. In addition, $\lim _{x_{1} \rightarrow \infty} f\left(x_{1}\right)=0$. This proves (C.22).

Open Access. This article is distributed under the terms of the Creative Commons Attribution License (CC-BY 4.0), which permits any use, distribution and reproduction in any medium, provided the original author(s) and source are credited.

\section{References}

[1] P. Kulish and E. Sklyanin, On solutions of the Yang-Baxter equation, Zap. Nauchn. Sem. LOMI 95 (1980) 129 [J. Soviet Math. 19 (1982) 1956].

[2] P. Kulish, Integrable graded magnets, Zap. Nauchn. Sem. LOMI 145 (1985) 140.

[3] J.H.H. Perk and C.L. Schultz, New families of commuting transfer matrices in $q$ state vertex models, Phys. Lett. A 84 (1981) 407 [InSPIRE].

[4] A. Alexandrov, V. Kazakov, S. Leurent, Z. Tsuboi and A. Zabrodin, Classical tau-function for quantum spin chains, JHEP 09 (2013) 064 [arXiv:1112.3310] [INSPIRE]. 
[5] V. Kazakov, S. Leurent and Z. Tsuboi, Baxter's Q-operators and operatorial Backlund flow for quantum (super)-spin chains, Commun. Math. Phys. 311 (2012) 787 [arXiv:1010.4022] [INSPIRE].

[6] A. Alexandrov, S. Leurent, Z. Tsuboi and A. Zabrodin, The master T-operator for the Gaudin model and the KP hierarchy, Nucl. Phys. B 883 (2014) 173 [arXiv:1306.1111] [INSPIRE].

[7] A. Zabrodin, Quantum Gaudin model and classical KP hierarchy, J. Phys. Conf. Ser. 482 (2014) 012047 [arXiv:1310.6985] [InSPIRE].

[8] A. Zabrodin, The master T-operator for vertex models with trigonometric R-matrices as classical tau-function, Theor. Math. Phys. 174 (2013) 52 [arXiv:1205.4152] [INSPIRE].

[9] A. Zabrodin, The Master T-Operator for Inhomogeneous XXX Spin Chain and mKP Hierarchy, SIGMA 10 (2014) 006 [arXiv:1310.6988] [INSPIRE].

[10] A. Zabrodin, Quantum spin chains and integrable many-body systems of classical mechanics, arXiv: 1409.4099 [INSPIRE].

[11] M. Sato and Y. Sato, Soliton equations as dynamical systems on infinite dimensional grassmann manifold, Lect. Notes Num. Appl. Anal. 5 (1982) 259.

[12] E. Date, M. Jimbo, M. Kashiwara and T. Miwa, Transformation groups for soliton equations, in Nonlinear integrable systems - classical and quantum, eds. M. Jimbo and T. Miwa, World Scientific (1983) pp. 39-120.

[13] M. Jimbo and T. Miwa, Solitons and infinite dimensional Lie algebras, Publ. RIMS 19 (1983) 943.

[14] I. Krichever, O. Lipan, P. Wiegmann and A. Zabrodin, Quantum integrable systems and elliptic solutions of classical discrete nonlinear equations, Commun. Math. Phys. 188 (1997) 267 [hep-th/9604080] [INSPIRE].

[15] A. Zabrodin, Hirota equation and the Bethe ansatz, Theor. Math. Phys. 116 (1998) 782.

[16] V. Kazakov, A.S. Sorin and A. Zabrodin, Supersymmetric Bethe ansatz and Baxter equations from discrete Hirota dynamics, Nucl. Phys. B 790 (2008) 345 [hep-th/0703147] [InSPIRE].

[17] A. Zabrodin, Backlund transformations for difference Hirota equation and supersymmetric Bethe ansatz, Theor. Math. Phys. 155 (2008) 567 [arXiv:0705.4006] [INSPIRE].

[18] I. Krichever and A. Zabrodin, Spin generalization of the Ruijsenaars-Schneider model, non-abelian 2D Toda chain and representations of Sklyanin algebra, Uspekhi Math. Nauk. 50 (1995) 3.

[19] P. Iliev, Rational Ruijsenaars-Schneider hierarchy and bispectral difference operators, Phys. D 229 (2007) 184 [math-ph/0609011].

[20] S.N.M. Ruijsenaars and H. Schneider, A New Class of Integrable Systems and Its Relation to Solitons, Annals Phys. 170 (1986) 370 [inSPIRE].

[21] N. Nekrasov, A. Rosly and S. Shatashvili, Darboux coordinates, Yang-Yang functional and gauge theory, Nucl. Phys. Proc. Suppl. 216 (2011) 69 [arXiv:1103.3919] [InSPIRE].

[22] D. Gaiotto and P. Koroteev, On Three Dimensional Quiver Gauge Theories and Integrability, JHEP 05 (2013) 126 [arXiv: 1304.0779] [INSPIRE].

[23] A. Gorsky, A. Zabrodin and A. Zotov, Spectrum of Quantum Transfer Matrices via Classical Many-Body Systems, JHEP 01 (2014) 070 [arXiv:1310.6958] [INSPIRE]. 
[24] S.N.M. Ruijsenaars, Action Angle Maps and Scattering Theory for Some Finite Dimensional Integrable Systems. 1. The Pure Soliton Case, Commun. Math. Phys. 115 (1988) 127 [INSPIRE].

[25] V. Fock, A. Gorsky, N. Nekrasov and V. Rubtsov, Duality in integrable systems and gauge theories, JHEP 07 (2000) 028.

[26] A. Baha Balantekin and I. Bars, Dimension and Character Formulas for Lie Supergroups, J. Math. Phys. 22 (1981) 1149 [INSPIRE].

[27] I. Bars, B. Morel and H. Ruegg, Kac-dynkin Diagrams and Supertableaux, J. Math. Phys. 24 (1983) 2253 [INSPIRE].

[28] M.D. Gould and R.B. Zhang, Classification Of All Star Irreps Of $g l(M \mid N)$, J. Math. Phys. 31 (1990) 2552 [inSPIRE].

[29] K. Hikami, P. Kulish and M. Wadati, Construction of integrable spin systems with long-range interaction, J. Phys. Soc. Japan 61 (1992) 3071.

[30] P.P. Kulish and N.Y. Reshetikhin, Diagonalization of $G L(N)$ invariant transfer matrices and quantum $N$ wave system (Lee model), J. Phys. A 16 (1983) L591 [inSPIRE].

[31] S. Belliard and É. Ragoucy, Nested Bethe ansatz for 'all' closed spin chains, J. Phys. A 41 (2008) 295202 [arXiv:0804.2822] [INSPIRE].

[32] H. Saleur, The Continuum limit of $s l(N / K)$ integrable superspin chains, Nucl. Phys. B 578 (2000) 552 [solv-int/9905007] [INSPIRE].

[33] É. Ragoucy and G. Satta, Analytical Bethe Ansatz for closed and open gl $(M \mid N)$ super-spin chains in arbitrary representations and for any Dynkin diagrams, JHEP 09 (2007) 001 [arXiv: 0706.3327] [INSPIRE].

[34] C.L. Schultz, Eigenvectors of the multicomponent generalization of the six-vertex model, Physica A 122 (1983) 71.

[35] V. Kazakov and P. Vieira, From characters to quantum (super)spin chains via fusion, JHEP 10 (2008) 050 [arXiv:0711.2470] [InSPIRE].

[36] I. Macdonald, Symmetric functions and Hall polynomials, 2nd ed., Oxford University Press, Oxford U.K. (1995).

[37] I.V. Cherednik, An analogue of character formula for Hecke algebras, Funct. Anal. Appl. 21 (1987) 94.

[38] I.V. Cherednik, Quantum groups as hidden symmetries of classic representation theory, in Proceedings of the XVII International Conference on Differential Geometric Methods in Theoretical Physics, A.I. Solomon ed., World Scientific, Singapore (1989) pg. 47-54.

[39] V. Bazhanov and N. Reshetikhin, Restricted Solid on Solid Models Connected With Simply Based Algebras and Conformal Field Theory, J. Phys. A 23 (1990) 1477 [InSPIRE].

[40] A. Kuniba, T. Nakanishi and J. Suzuki, Functional relations in solvable lattice models. 1: Functional relations and representation theory, Int. J. Mod. Phys. A 9 (1994) 5215 [hep-th/9309137] [INSPIRE].

[41] A. Kuniba, Y. Ohta and J. Suzuki, Quantum Jacobi-Trudi and Giambelli formulae for $U_{q}\left(B_{r}^{(1)}\right)$ from analytic Bethe ansatz, J. Phys. A 28 (1995) 6211 [hep-th/9506167] [INSPIRE].

[42] Z. Tsuboi, Analytic Bethe ansatz and functional equations for Lie superalgebra $s l(r+1 \mid s+1), J$. Phys. A 30 (1997) 7975 [arXiv:0911.5386] [INSPIRE]. 
[43] Z. Tsuboi, Analytic Bethe Ansatz And Functional Equations Associated With Any Simple Root Systems Of The Lie Superalgebra sl $(r+1 \mid s+1)$, Physica A 252 (1998) 565 [arXiv:0911.5387] [INSPIRE].

[44] A. Hegedus, Discrete Hirota dynamics for AdS/CFT, Nucl. Phys. B 825 (2010) 341 [arXiv:0906.2546] [INSPIRE].

[45] N. Gromov, V. Kazakov and Z. Tsuboi, PSU $(2,2 \mid 4)$ Character of Quasiclassical AdS/CFT, JHEP 07 (2010) 097 [arXiv: 1002.3981] [INSPIRE].

[46] V.V. Bazhanov and Z. Tsuboi, Baxter's Q-operators for supersymmetric spin chains, Nucl. Phys. B 805 (2008) 451 [arXiv:0805.4274] [INSPIRE].

[47] A. Berele and A. Regev, Hook Young-Diagrams With Applications To Combinatorics And To Representations Of Lie-Superalgebras, Adv. Math. 64 (1987) 118 [INSPIRE].

[48] A. Orlov and T. Shiota, Schur function expansion for normal matrix model and associated discrete matrix models, Phys. Lett. A 343 (2005) 384.

[49] V. Enolski and J. Harnad, Schur function expansions of KP tau functions associated to algebraic curves, Russ. Math. Surveys 6 (2011) 767 [arXiv:1012.3152] [InSPIRE].

[50] N. Gromov, V. Kazakov and P. Vieira, Exact Spectrum of Anomalous Dimensions of Planar $N=4$ Supersymmetric Yang-Mills Theory, Phys. Rev. Lett. 103 (2009) 131601 [arXiv:0901.3753] [INSPIRE].

[51] N. Gromov, V. Kazakov, S. Leurent and Z. Tsuboi, Wronskian Solution for AdS/CFT Y-system, JHEP 01 (2011) 155 [arXiv: 1010.2720] [INSPIRE].

[52] Z. Tsuboi, Wronskian solutions of the T, $Q$ and $Y$-systems related to infinite dimensional unitarizable modules of the general linear superalgebra $g l(M \mid N)$, Nucl. Phys. B 870 (2013) 92 [arXiv:1109.5524] [InSPIRE].

[53] Y. Shigyo, On addition formulae of KP, mKP and BKP hierarchies, SIGMA 9 (2013) 35 [arXiv: 1212.1952].

[54] I.M. Krichever, Rational solutions of the Kadomtsev Petviashvili equation and integrable systems of $N$ particles on a line, Funct. Anal. Appl. 12 (1978) 59.

[55] I.M. Krichever, Rational solutions of the Zakharov-Shabat equations and completely integrable systems of $N$ particles on a line, J. Sov. Math. 21 (1983) 335.

[56] T. Shiota, Calogero-Moser hierarchy and KP hierarchy, J. Math. Phys. 35 (1994) 5844 [hep-th/9402021] [INSPIRE].

[57] E. Mukhin, V. Tarasov and A. Varchenko, Gaudin Hamiltonians generate the Bethe algebra of a tensor power of vector representation of $g l_{N}$, St. Petersburg Math. J. 22 (2011) 463 [arXiv:0904.2131].

[58] E. Mukhin, V. Tarasov and A. Varchenko, KZ characteristic variety as the zero set of classical Calogero-Moser Hamiltonians, SIGMA 8 (2012) 72 [arXiv:1201.3990].

[59] E. Mukhin, V. Tarasov and A. Varchenko, Bethe subalgebras of the group algebra of the symmetric group, arXiv: 1004.4248 [INSPIRE].

[60] E. Mukhin, V. Tarasov and A. Varchenko, Spaces of quasi-exponentials and representations of the Yangian $Y\left(g l_{N}\right)$, arXiv:1303.1578.

[61] G. Wilson, Collisions of Calogero-Moser particles and an adelic Grassmannian, Invent. Math. 133 (1998) 1.

[62] A. Givental and B. Kim, Quantum cohomology of flag manifolds and Toda lattices, Commun. Math. Phys. 168 (1995) 609 [hep-th/9312096] [INSPIRE]. 